\title{
Analysis of Transient and Steady-State Behavior of a Multichannel Filtered- $x$ Partial-Error Affine Projection Algorithm
}

\author{
Alberto Carini ${ }^{1}$ and Giovanni L. Sicuranza ${ }^{2}$ \\ ${ }^{1}$ Information Science and Technology Institute, University of Urbino "Carlo Bo", 61029 Urbino, Italy \\ ${ }^{2}$ Department of Electrical, Electronic and Computer Engineering, University of Trieste, 34127 Trieste, Italy
}

Received 28 April 2006; Revised 24 November 2006; Accepted 27 November 2006

Recommended by Kutluyil Dogancay

The paper provides an analysis of the transient and the steady-state behavior of a filtered- $x$ partial-error affine projection algorithm suitable for multichannel active noise control. The analysis relies on energy conservation arguments, it does not apply the independence theory nor does it impose any restriction to the signal distributions. The paper shows that the partial-error filtered- $x$ affine projection algorithm in presence of stationary input signals converges to a cyclostationary process, that is, the mean value of the coefficient vector, the mean-square error and the mean-square deviation tend to periodic functions of the sample time.

Copyright (c) 2007 A. Carini and G. L. Sicuranza. This is an open access article distributed under the Creative Commons Attribution License, which permits unrestricted use, distribution, and reproduction in any medium, provided the original work is properly cited.

\section{INTRODUCTION}

Active noise controllers are based on the destructive interference in given locations of the noise produced by some primary sources and the interfering signals generated by some secondary sources driven by an adaptive controller [1]. A commonly used strategy is based on the so-called feedforward methods, where some reference signals measured in the proximity of the noise source are available. These signals are used together with the error signals captured in the proximity of the zone to be silenced in order to adapt the controller. Single-channel and multichannel schemes have been proposed in the literature according to the number of reference sensors, error sensors, and secondary sources used. A single-channel active noise controller makes use of a single reference sensor, actuator, and error sensor and it gives, in principle, attenuation of the undesired disturbance in the proximity of the point where the error sensor is located. In the multichannel approach, in order to spatially extend the silenced region, multiple reference sensors, actuators and error sensors are used. Due to the multiplicity of the signals involved, to the strong correlations between them and to the long impulse response of the acoustic paths, multichannel active noise controllers suffer the complexity of the coefficient updates, the data storage requirements, and the slow convergence of the adaptive algorithms [2]. To improve the convergence speed, different filtered $x$ affine projection (FXAP) algorithms have been used $[3,4]$ in place of the usual filtered- $x$ LMS algorithms, but at the expense of a further, even though limited, increment of the complexity of updates. Various techniques have been proposed in the literature to keep low the implementation complexity of adaptive FIR filters having long impulse responses. Most of them can be usefully applied to the filtered- $x$ algorithms, too, especially in the multichannel situations. A first approach is based on the so-called interpolated FIR filters [5], where a few impulse response samples are removed and then their values are derived using some type of interpolation scheme. However, the success of this implementation is based on the hypothesis that practical FIR filters have an impulse response with a smooth predictable envelope, which is not applicable to the acoustic paths. Another approach is based on data-selective updates which are sparse in time. This approach can be suitably described in the framework of the set-membership filtering (SMF) where a filter is designed to achieve a specified bound on the magnitude of the output error [6]. Finally, a set of well-established techniques is based on selective partial updates (PU) where selected blocks of filter coefficients are updated at every iteration in a sequential or periodic manner [7] or by using an appropriate selection criterion [8]. Among 
the partial update strategies, a simple yet effective approach is provided by the partial error (PE) technique, which has been first applied in [7] for reducing the complexity of linear multichannel controllers equipped with the filtered- $x$ LMS algorithm. The PE technique consists in using sequentially at each iteration only one of the $K$ error sensor signals in place of their combination and it is capable to reduce the adaptation complexity with a factor $K$. In [9], the PE technique was applied, together with other methods, for reducing the computational load of multichannel active noise controllers equipped with filtered- $x$ affine projection (AP) algorithms. When dealing with novel adaptive filters, it is important to assess their performance not only through extensive simulations but also with theoretical analysis results. In the literature, very few results deal with the analysis of filtered- $x$, affine projection or partial-update algorithms. The convergence analysis results for these algorithms are often based on the independence theory (IT) and they constrain the probability distribution of the input signal to be Gaussian or spherically invariant [10]. The IT hypothesis assumes statistical independence of time-lagged input data vectors. As it is too strong for filtered- $x$ LMS [11] and AP algorithms [12], different approaches have been studied in the literature in order to overcome this hypothesis. In [11], an analysis of the mean weight behavior of the filtered- $x$ LMS algorithm, based only on neglecting the correlation between coefficient and signal vectors, is presented. Moreover, the analysis of [11] does not impose any restriction on the signal distributions. Another analysis approach that avoids IT is applied in [12] for the mean-square performance analysis of AP algorithms. This relies on energy conservation arguments, and no restriction is imposed on the signal distributions. In [4], we applied and adapted the approach of [12] for analyzing the convergence behavior of multichannel FX-AP algorithms. In this paper, we extend the analysis approach of [4] and study the transient and steady-state behavior of a filtered- $x$ partial error affine projection (FX-PE-AP) algorithm. The paper shows that the FX-PE-AP algorithm in presence of stationary input signals converges to a cyclostationary process, that is, that the mean value of the coefficient vector, the mean-square-error, and the mean-square-deviation tend to periodic functions of the sample time. We also show the FX-PE-AP algorithm is capable to reduce the adaptation complexity with a factor $K$ with respect to an approximate FX-AP algorithm introduced in [4], but it also reduces the convergence speed by the same factor.

The paper is organized as follows. Section 2 reviews the multichannel feedforward active noise controller structure and introduces the FX-PE-AP algorithm. Section 3 discusses the asymptotic solution of the FX-PE-AP algorithm and compares it with that of FX-AP algorithms and with the minimum-mean-square solution of the ANC problem. Section 4 presents the analysis of the transient and steady-state behavior of the FX-PE-AP algorithm. Section 5 provides some experimental results. Conclusions follow in Section 6.

Throughout this paper, small boldface letters are used to denote vectors and bold capital letters are used to denote ma- trices, for example, $\mathbf{x}$ and $\mathbf{X}$, all vectors are column vectors, the boldface symbol I indicates an identity matrix of appropriate dimensions, the symbol $\odot$ denotes linear convolution, $\operatorname{diag}\{\cdots\}$ is a block-diagonal matrix of the entries, $E[\cdot]$ denotes mathematical expectation, $\|\cdot\|_{\Sigma}^{2}$ is the weighted Euclidean norm, for example, $\|\mathbf{w}\|_{\Sigma}^{2}=\mathbf{w}^{T} \mathbf{\Sigma} \mathbf{w}$ with $\boldsymbol{\Sigma}$ a symmetric positive definite matrix, $\operatorname{vec}\{\cdot\}$ indicates the vector operator and $\operatorname{vec}^{-1}\{\cdot\}$ the inverse vector operator that returns a square matrix from an input vector of appropriate dimensions, $\otimes$ denotes the Kronecker product, $a \% b$ is the remainder of the division of $a$ by $b$, and $|a|$ is the absolute value of $a$.

\section{THE PARTIAL-ERROR FILTERED- $x$ AP ALGORITHM}

The schematic description of a multichannel feedforward active noise controller (ANC) is provided in Figure 1. I reference sensors collect the corresponding input signals from the noise sources and $K$ error sensors collect the error signals at the interference locations. The signals coming from these sensors are used by the controller in order to adaptively estimate $J$ output signals which feed $J$ actuators. The corresponding block diagram is reported in Figure 2. The propagation of the original noise up to the region to be silenced is described by the transfer functions $p_{k, i}(z)$ representing the primary paths. The secondary noise signals propagate through secondary paths, which are characterized by the transfer functions $s_{k, j}(z)$. We assume there is no feedback between loudspeakers and reference sensors. The primary source signals filtered by the impulse responses of the secondary paths model, with transfer functions $\widetilde{s}_{k, j}(z)$, are used for the adaptive filter update, and for this reason the adaptation algorithm is called filtered- $x$. Figure 2 illustrates also the delay-compensation scheme [13] that is used throughout the paper. To compensate for the propagation delay introduced by the secondary paths, the output of the primary paths $\mathbf{d}(n)$ is estimated with $\widetilde{\mathbf{d}}(n)$ by subtracting the output of the secondary paths model from the error sensors signals $\mathbf{d}(n)$, and the error signal $\widetilde{\mathbf{e}}(n)$ between $\widetilde{\mathbf{d}}(n)$ and the output of the adaptive filter is used for the adaptation of the filter $\mathbf{w}(n)$. A copy of this filter is used for the actuators' output estimation.

Preliminary and independent evaluations of the secondary paths transfer functions are needed. For generality purposes, the theoretical results we present assume imperfect modelling of the secondary paths (we consider $\tilde{s}_{k, j}(z) \neq$ $s_{k, j}(z)$ for any choice of $j$ and $k$ ), but all the results hold also for perfect modelling (i.e., for $\widetilde{s}_{k, j}(z)=s_{k, j}(z)$ ). Indeed, the experimental results of Section 5 refer to ANC systems with perfect modelling of the secondary paths. When necessary, we will highlight in the paper the different behavior of the system under perfect and imperfect estimations of the secondary paths.

Very mild assumptions are posed in this paper on the adaptive controller. Indeed, we assume that any input $i$ of the controller is connected to any output $j$ through a filter whose output depends linearly on the filter coefficients, that is, we assume that the $j$ th actuator output is given by the following 


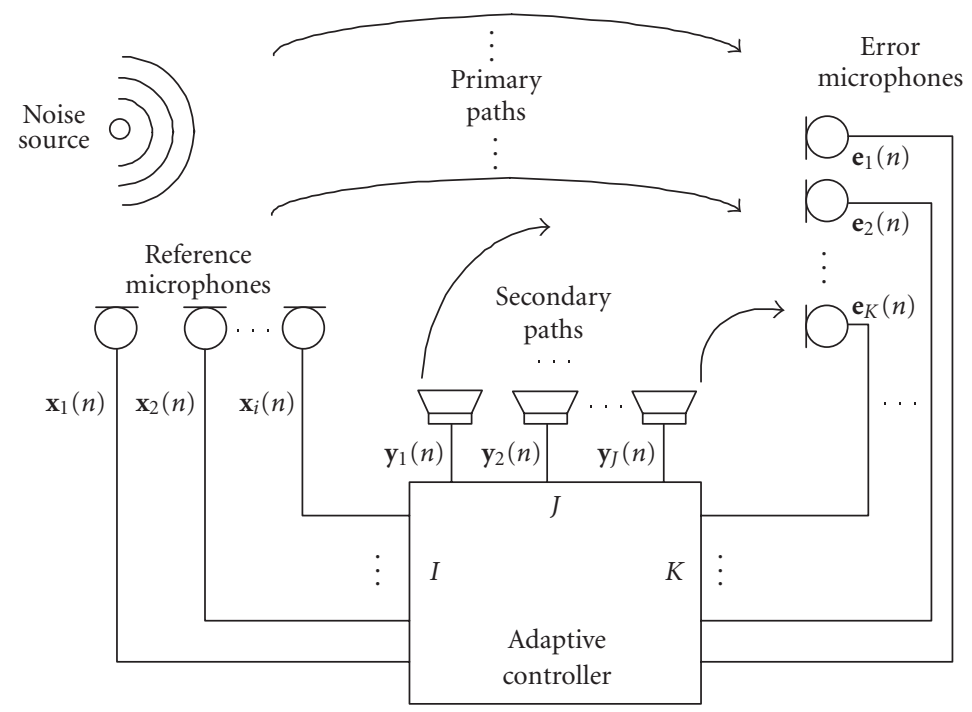

FIGURE 1: A schematic description of multichannel feedforward active noise control.

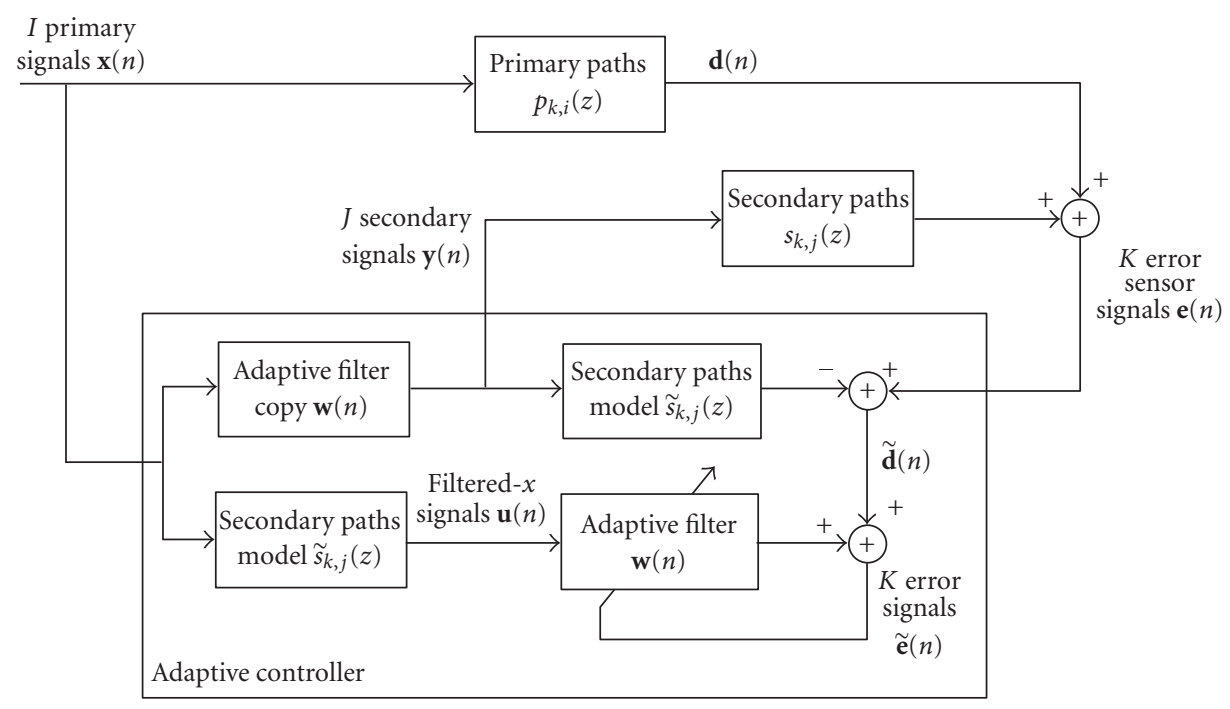

FIGURE 2: Delay-compensated filtered- $x$ structure for active noise control.

vector equation:

$$
y_{j}(n)=\sum_{i=1}^{I} \mathbf{x}_{i}^{T}(n) \mathbf{w}_{j, i}(n),
$$

where $\mathbf{w}_{j, i}(n)$ is the coefficient vector of the filter that connects the input $i$ to the output $j$ of the adaptive controller, and $\mathbf{x}_{i}(n)$ is the $i$ th primary source input signal vector. In particular, $\mathbf{x}_{i}(n)$ is here expressed as a vector function of the signal samples $x_{i}(n)$ whose general form is given by

$$
\mathbf{x}_{i}(n)=\left[f_{1}\left[x_{i}(n)\right], f_{2}\left[x_{i}(n)\right], \ldots, f_{N}\left[x_{i}(n)\right]\right]^{T},
$$

where $f_{i}[\cdot]$, for any $i=1, \ldots, N$, is a time-invariant functional of its argument. Equations (1) and (2) include lin- ear filters, truncated Volterra filters of any order $p$ [14], radial basis function networks [15], filters based on functional expansions [16], and other nonlinear filter structures. In Section 5 we provide experimental results for linear filters, where the vector $\mathbf{x}_{i}(n)$ reduces to

$$
\mathbf{x}_{i}(n)=\left[x_{i}(n), x_{i}(n-1), \ldots, x_{i}(n-N+1)\right]^{T},
$$

and for filters based on a piecewise linear functional expansion with the vector $\mathbf{x}_{i}(n)$ given by

$$
\begin{aligned}
\mathbf{x}_{i}(n)= & {\left[x_{i}(n), x_{i}(n-1), \ldots, x_{i}(n-N+1),\right.} \\
& \left.\left|x_{i}(n)-a\right|, \ldots,\left|x_{i}(n-N+1)-a\right|\right]^{T},
\end{aligned}
$$

where $a$ is an appropriate constant. 
To introduce the PE-FX-AP algorithm analyzed in subsequent sections, we make use of quantities defined in Table 1.

Our objective is to estimate the coefficient vector $\mathbf{w}_{o}=$ $\left[\mathbf{w}_{1}^{T}, \mathbf{w}_{2}^{T}, \ldots, \mathbf{w}_{J}^{T}\right]^{T}$ that minimizes the cost function given in

$$
J_{o}=E\left[\sum_{k=1}^{K}\left(d_{k}(n)+\sum_{j=1}^{J} s_{k, j}(n) \odot\left(\mathbf{w}_{j}^{T} \mathbf{x}(n)\right)\right)^{2}\right] .
$$

Several adaptive filters have been proposed in the literature to estimate the filter $\mathbf{w}_{o}$. In [4], we have analyzed the convergence properties of the approximate FX-AP algorithm with adaptation rule given by

$$
\mathbf{w}(n+1)=\mathbf{w}(n)-\mu \sum_{k=0}^{K} \widetilde{\mathbf{U}}_{k}(n) \widetilde{\mathbf{R}}_{k}^{-1}(n) \tilde{\mathbf{e}}_{k}(n),
$$

where

$$
\widetilde{\mathbf{R}}_{k}(n)=\tilde{\mathbf{U}}_{k}^{T}(n) \tilde{\mathbf{U}}_{k}(n)+\delta \mathbf{I}
$$

In this paper, we consider the FX-PE-AP algorithm characterized by the adaptation rule of

$$
\mathbf{w}(n+1)=\mathbf{w}(n)-\mu \widetilde{\mathbf{U}}_{n \% K}(n) \widetilde{\mathbf{R}}_{n \% K}^{-1}(n) \widetilde{\mathbf{e}}_{n \% K}(n),
$$

where $n \% K$ is the remainder of the division of $n$ by $K$. The adaptation rule in (8) has been obtained by applying the PE methodology to the approximate FX-AP algorithm of (6). At each iteration, only one of the $K$ error sensor signals is used for the controller adaptation. The error sensor signal employed for the adaptation is chosen with a round-robin strategy. Thus, compared with (6), the FX-PE-AP adaptation in (8) reduces the computational load by a factor $K$.

The exact value of the estimated residual error $\tilde{e}_{k}(n)$ is given by

$$
\begin{aligned}
\tilde{e}_{k}(n)= & d_{k}(n)+\sum_{j=1}^{J}\left(s_{k, j}(n)-\widetilde{s}_{k, j}(n)\right) \odot\left(\mathbf{w}_{j}^{T}(n) \mathbf{x}(n)\right) \\
& +\sum_{j=1}^{J} \mathbf{w}_{j}^{T}(n) \widetilde{\mathbf{u}}_{k, j}(n) .
\end{aligned}
$$

In order to analyze the FX-PE-AP algorithm, we introduce in (9) the approximation

$$
\begin{aligned}
& \sum_{j=1}^{J}\left(s_{k, j}(n)-\tilde{s}_{k, j}(n)\right) \odot\left(\mathbf{w}_{j}^{T}(n) \mathbf{x}(n)\right) \\
& \quad \cong \sum_{j=1}^{J} \mathbf{w}_{j}^{T}(n) \cdot\left(\left(s_{k, j}(n)-\tilde{s}_{k, j}(n)\right) \odot \mathbf{x}(n)\right),
\end{aligned}
$$

which allows us to simplify (9) and to obtain

$$
\tilde{e}_{k}(n)=d_{k}(n)+\sum_{j=1}^{J} \mathbf{w}_{j}^{T}(n) \mathbf{u}_{k, j}(n) .
$$

Note that the expression in (11) is correct when we perfectly estimate the secondary paths or when $\mathbf{w}(n)$ is constant, that is, when we work with small step-size values. On the contrary, the expression in (11) is only an approximation for large step-sizes and in presence of secondary path estimation errors, but it allows an insightful analysis of the effects of these estimation errors.

By introducing the result of (11) in (8), we obtain the following equation:

$$
\begin{aligned}
\mathbf{w}(n+1)= & \mathbf{w}(n)-\mu \widetilde{\mathbf{U}}_{n \% K}(n) \widetilde{\mathbf{R}}_{n \% K}^{-1}(n) \\
& \times\left(\mathbf{d}_{n \% K}(n)+\mathbf{U}_{n \% K}^{T}(n) \mathbf{w}(n)\right),
\end{aligned}
$$

which can also be written in the compact form of

$$
\mathbf{w}(n+1)=\mathbf{V}_{n \% K}(n) \mathbf{w}(n)-\mathbf{v}_{n \% K}(n),
$$

with

$$
\begin{aligned}
& \mathbf{V}_{k}(n)=\mathbf{I}-\mu \widetilde{\mathbf{U}}_{k}(n) \widetilde{\mathbf{R}}_{k}^{-1}(n) \mathbf{U}_{k}^{T}(n), \\
& \mathbf{v}_{k}(n)=\mu \widetilde{\mathbf{U}}_{k}(n) \widetilde{\mathbf{R}}_{k}^{-1}(n) \mathbf{d}_{k}(n)
\end{aligned}
$$

By iterating $K$ times (13) from $n=m K+i$ till $n=m K+$ $i+K-1$, with $m \in \mathbb{N}$ and $0 \leq i<K$, we obtain the expression of (15), which will be used for the algorithm analysis,

$$
\mathbf{w}(m K+i+K)=\mathbf{M}_{i}(m K+i) \mathbf{w}(m K+i)-\mathbf{m}_{i}(m K+i),
$$

where

$$
\begin{aligned}
\mathbf{M}_{i}(n)= & \mathbf{V}_{(i+K-1) \% K}(n+K-1) \mathbf{V}_{(i+K-2) \% K}(n+K-2) \\
& \times \cdots \mathbf{V}_{i \% K}(n), \\
\mathbf{m}_{i}(n)= & \mathbf{V}_{(i+K-1) \% K}(n+K-1) \cdots \mathbf{V}_{(i+1) \% K}(n+1) \mathbf{v}_{i \% K}(n) \\
+ & \mathbf{V}_{(i+K-1) \% K}(n+K-1) \cdots \mathbf{V}_{(i+2) \% K}(n+2) \\
& \times \mathbf{V}_{(i+1) \% K}(n+1) \\
& +\cdots+\mathbf{v}_{(i+K-1) \% K}(n+K-1) .
\end{aligned}
$$

\section{THE ASYMPTOTIC SOLUTION}

For $i$ ranging from 0 to $K-1$, (15) provides a set of $K$ independent equations that can be separately studied. The system matrix $\mathbf{M}_{i}(n)$ and excitation matrix $\mathbf{m}_{i}(n)$ have different statistical properties for different indexes $i$. For every $i$, the recursion in (15) converges to a different asymptotic coefficient vector and it provides different values of the steadystate mean-square error and the mean-square deviation. If the input signals are stationary and if the recursion in (15) is convergent for every $i$, it can be shown that the algorithm converges to a cyclostationary process of periodicity $K$.

For every index $i$, the coefficient vector $\mathbf{w}(m K+i)$ tends for $m \rightarrow+\infty$ to an asymptotic vector $\mathbf{w}_{\infty, i}$, which depends on the statistical properties of the input signals. In fact, by taking the expectation of (15) and considering the fixed point of this equation, it can be easily deduced that

$$
\mathbf{w}_{\infty, i}=\left(E\left[\mathbf{M}_{i}(n)\right]-\mathbf{I}\right)^{-1} E\left[\mathbf{m}_{i}(n)\right] .
$$


TABle 1: Quantities used for the algorithms definition.

\begin{tabular}{|c|c|c|}
\hline Quantity & Dimensions & Description \\
\hline$I$ & 1 & Number of primary source signals. \\
\hline$J$ & 1 & Number of secondary source signals. \\
\hline K & 1 & Number of error sensors. \\
\hline$L$ & 1 & AP order. \\
\hline$N$ & 1 & Number of elements of vectors $\mathbf{x}_{i}(n)$ and $\mathbf{w}_{j, i}(n)$. \\
\hline$M=N \cdot I \cdot J$ & 1 & Number of coefficients of $\mathbf{w}(n)$ \\
\hline$s_{k, j}(n)$ & 1 & $\begin{array}{l}\text { Impulse response of the secondary path that connects } \\
\text { the } j \text { th secondary source to the } k \text { th error sensor. }\end{array}$ \\
\hline$\tilde{s}_{k, j}(n)$ & 1 & $\begin{array}{l}\text { Estimated secondary path impulse response from the } \\
j \text { th secondary source to the } k \text { th error sensor. }\end{array}$ \\
\hline $\mathbf{x}_{i}(n)$ & $N \times 1$ & ith primary source input signal vector. \\
\hline $\mathbf{x}(n)=\left[\mathbf{x}_{1}^{T}(n), \ldots, \mathbf{x}_{I}^{T}(n)\right]^{T}$ & $N \cdot I \times 1$ & Full primary source input signal vector. \\
\hline $\mathbf{w}_{j, i}(n)$ & $N \times 1$ & $\begin{array}{l}\text { Coefficient vector of the filter that connects the input } i \\
\text { to the output } j \text { of the ANC. }\end{array}$ \\
\hline $\mathbf{w}_{j}(n)=\left[\mathbf{w}_{j, 1}^{T}(n), \ldots, \mathbf{w}_{j, I}^{T}(n)\right]^{T}$ & $N \cdot I \times 1$ & $\begin{array}{l}\text { Aggregate of the coefficient vectors related to the output } \\
j \text { of ANC. }\end{array}$ \\
\hline $\mathbf{w}(n)=\left[\mathbf{w}_{1}^{T}(n), \ldots, \mathbf{w}_{I}^{T}(n)\right]^{T}$ & $M \times 1$ & Full coefficient vector of ANC. \\
\hline$y_{j}(n)=\mathbf{w}_{j}^{T}(n) \mathbf{x}(n)$ & 1 & jth secondary source signal. \\
\hline$d_{k}(n)$ & 1 & Output of the $k$ th primary path. \\
\hline $\mathbf{d}_{k}(n)=\left[d_{k}(n), \ldots, d_{k}(n-L+1)\right]^{T}$ & $L \times 1$ & Vector of the $L$ past outputs of the $k$ th primary path. \\
\hline $\mathbf{d}(n)=\left[\mathbf{d}_{1}^{T}(n), \ldots, \mathbf{d}_{K}^{T}(n)\right]^{T}$ & $L \cdot K \times 1$ & Full vector of the $L$ past outputs of the primary paths. \\
\hline$\tilde{d}_{k}(n)=d_{k}(n)+\sum_{j=1}^{J}\left(s_{k, j}(n)-\tilde{s}_{k, j}(n)\right) \odot y_{j}(n)$ & 1 & Estimated output of the $k$ th primary path. \\
\hline $\mathbf{u}_{k, j}(n)=s_{k, j}(n) \odot \mathbf{x}(n)$ & $N \cdot I \times 1$ & $\begin{array}{l}\text { Filtered- } x \text { vector obtained by filtering, sample by } \\
\text { sample, } \mathbf{x}(n) \text { with } s_{k, j}(n)\end{array}$ \\
\hline $\mathbf{u}_{k}(n)=\left[\mathbf{u}_{k, 1}^{T}(n), \ldots, \mathbf{u}_{k, J}^{T}(n)\right]^{T}$ & $M \times 1$ & Aggregate of the filtered- $x$ vectors associated with output $k$. \\
\hline $\mathbf{U}_{k}(n)=\left[\mathbf{u}_{k}(n), \mathbf{u}_{k}(n-1), \ldots, \mathbf{u}_{k}(n-L+1)\right]$ & $M \times L$ & Matrix constituted by the last $L$ filtered- $x$ vectors $\mathbf{u}_{k}(n)$. \\
\hline$\tilde{\mathbf{u}}_{k, j}(n)=\widetilde{s}_{k, j}(n) \odot \mathbf{x}(n)$ & $N \cdot I \times 1$ & $\begin{array}{l}\text { Filtered- } x \text { vector obtained by filtering, sample by } \\
\text { sample, } \mathbf{x}(n) \text { with } \widetilde{s}_{k, j}(n)\end{array}$ \\
\hline$\tilde{\mathbf{u}}_{k}(n)=\left[\tilde{\mathbf{u}}_{k, 1}^{T}(n), \ldots, \widetilde{\mathbf{u}}_{k, J}^{T}(n)\right]^{T}$ & $M \times 1$ & $\begin{array}{l}\text { Aggregate of the filtered- } x \text { vectors associated with } \\
\text { estimated output } k \text {. }\end{array}$ \\
\hline$\tilde{\mathbf{U}}_{k}(n)=\left[\tilde{\mathbf{u}}_{k}(n), \tilde{\mathbf{u}}_{k}(n-1), \ldots, \tilde{\mathbf{u}}_{k}(n-L+1)\right]$ & $M \times L$ & Matrix constituted by the last $L$ filtered- $x$ vectors $\tilde{\mathbf{u}}_{k}(n)$ \\
\hline$\tilde{e}_{k}(n)=\tilde{d}_{k}(n)+\sum_{j=1}^{J} \tilde{\mathbf{u}}_{k, j}^{T}(n) \mathbf{w}_{j}(n)$ & 1 & $k$ th error signal. \\
\hline$\tilde{\mathbf{e}}_{k}(n)=\left[\tilde{e}_{k}(n), \ldots, \tilde{e}_{k}(n-L+1)\right]^{T}$ & $L \times 1$ & Vector of $L$ past errors on $k$ th primary path. \\
\hline$\tilde{\mathbf{e}}(n)=\left[\widetilde{\mathbf{e}}_{1}^{T}(n), \ldots, \widetilde{\mathbf{e}}_{K}^{T}(n)\right]^{T}$ & $L \cdot K \times 1$ & Full vector of errors. \\
\hline
\end{tabular}

Since the matrices $E\left[\mathbf{M}_{i}(n)\right]$ and $\left[\mathbf{m}_{i}(n)\right]$ vary with $i$, so do the asymptotic coefficient vectors $\mathbf{w}_{\infty, i}$. Thus, the vector $\mathbf{w}(n)$ for $n \rightarrow+\infty$ tends to the periodic sequence formed by the repetition of the $K$ vectors $\mathbf{w}_{\infty, i}$ with $i=0,1, \ldots, K-1$.

The asymptotic sequence varies with the step-size $\mu$ and with the estimation errors $\widetilde{s}_{k, j}(z)-s_{k, j}(z)$ of the secondary paths. As we already observed for FX-AP algorithms [4], the asymptotic solution in (18) differs from the minimummean-square (MMS) solution of the active noise control problem, which is given by (19) [17],

$$
\mathbf{w}_{o}=-\mathbf{R}_{u u}^{-1} \mathbf{R}_{u d}
$$


where $\mathbf{R}_{u u}$ and $\mathbf{R}_{u d}$ are defined, respectively, in

$$
\begin{aligned}
& \mathbf{R}_{u u}=E\left[\sum_{k=1}^{K} \mathbf{u}_{k}(n) \mathbf{u}_{k}^{T}(n)\right], \\
& \mathbf{R}_{u d}=E\left[\sum_{k=1}^{K} \mathbf{u}_{k}(n) d_{k}(n)\right] .
\end{aligned}
$$

Moreover, $\mathbf{w}_{\infty, i}$ for every $i$ differs also from the asymptotic solution $\mathbf{w}_{\infty}$ of the adaptation rule in (6), which is given by [4]

$$
\begin{aligned}
\mathbf{w}_{\infty}= & -E\left[\sum_{k=1}^{K} \tilde{\mathbf{U}}_{k}(n) \widetilde{\mathbf{R}}_{k}^{-1}(n) \mathbf{U}_{k}^{T}(n)\right]^{-1} \\
& \times E\left[\sum_{k=1}^{K} \tilde{\mathbf{U}}_{k}(n) \widetilde{\mathbf{R}}_{k}^{-1}(n) \mathbf{d}_{k}(n)\right] .
\end{aligned}
$$

Nevertheless, when $\mu$ tends to 0 , the vectors $\mathbf{w}_{\infty, i}$ tend to the same asymptotic solution $\mathbf{w}_{\infty}$ of (6). In fact, it can be verified that the expression in (18), when $\mu$ tends to 0 , converges to the following expression:

$$
\begin{aligned}
& \mathbf{w}_{\infty, i}=-E\left[\sum_{k=1}^{K} \widetilde{\mathbf{U}}_{(i+K-k) \% K}(n+K-k) \widetilde{\mathbf{R}}_{(i+K-k) \% K}^{-1}(n+K-k)\right. \\
&\left.\times \mathbf{U}_{(i+K-k) \% K}^{T}(n+K-k)\right]^{-1} \\
& \times E\left[\sum_{k=1}^{K} \widetilde{\mathbf{U}}_{(i+K-k) \% K}(n+K-k) \widetilde{\mathbf{R}}_{(i+K-k) \% K}^{-1}(n+K-k)\right. \\
& \\
&\left.\times \mathbf{d}_{(i+K-k) \% K}(n+K-k)\right]
\end{aligned}
$$

which in the hypothesis of stationary input signals is equal to the expression in (21).

\section{TRANSIENT ANALYSIS AND STEADY- STATE ANALYSIS}

The transient analysis aims to study the time evolution of the expectation of the weighted Euclidean norm of the coefficient vector $E\left[\|\mathbf{w}(n)\|_{\boldsymbol{\Sigma}}^{2}\right]=\mathbf{w}(n)^{T} \mathbf{\Sigma} \mathbf{w}(n)$ for some choices of the symmetric positive definite matrix $\Sigma$ [12]. Moreover, the limit for $n \rightarrow+\infty$ of the same quantity, again for some appropriate choices of the matrix $\Sigma$, is needed for the steadystate analysis. For simplicity, in the following we assume to work with stationary input signals and, according to (15), we separately analyze the evolution of $E\left[\|\mathbf{w}(m K+i)\|_{\Sigma}^{2}\right]$ for the different indexes $i$.

\subsection{Energy conservation relation}

We first derive a recursive relation for $\|\mathbf{w}(m K+i)\|_{\Sigma}^{2}$. By substituting the expression of (15) in the definition of $\| \mathbf{w}(m K+$ $i+K) \|_{\Sigma}^{2}$, we obtain the relation of

$$
\begin{aligned}
\|\mathbf{w}(m K+i+K)\|_{\Sigma}^{2}= & \mathbf{w}^{T}(m K+i+K) \mathbf{\Sigma} \mathbf{w}(m K+i+K) \\
= & \mathbf{w}^{T}(m K+i) \boldsymbol{\Sigma}^{\prime}{ }_{i}(m K+i) \mathbf{w}(m K+i) \\
& -2 \mathbf{w}^{T}(m K+i) \mathbf{q}_{\boldsymbol{\Sigma}, i}(m K+i) \\
& +\mathbf{m}_{i}^{T}(m K+i) \mathbf{\Sigma} \mathbf{m}_{i}(m K+i),
\end{aligned}
$$

where we have introduced the quantities $\boldsymbol{\Sigma}_{i}^{\prime}(n)$ and $\mathbf{q}_{\boldsymbol{\Sigma}, i}(n)$ which are defined, respectively, in

$$
\begin{aligned}
\boldsymbol{\Sigma}_{i}^{\prime}(n) & =\mathbf{M}_{i}^{T}(n) \mathbf{\Sigma} \mathbf{M}_{i}(n), \\
\mathbf{q}_{\boldsymbol{\Sigma}, i}(n) & =\mathbf{M}_{i}^{T}(n) \boldsymbol{\Sigma} \mathbf{m}_{i}(n) .
\end{aligned}
$$

Equation (23) provides an energy conservation relation, which is the basis of our analysis. The relation of (23) has the same role of the energy conservation relation employed in [12]. No approximation has been used for deriving the expression of (23).

\subsection{Transient analysis}

We are now interested in studying the time evolution of $E\left[\|\mathbf{w}(m K+i)\|_{\Sigma}^{2}\right]$ where $\boldsymbol{\Sigma}$ is a symmetric and positive definite square matrix. For this purpose, we follow the approach of $[12,18,19]$.

In the analysis of filtered- $x$ and AP algorithms, it is common to assume $\mathbf{w}(n)$ to be uncorrelated with some functions of the filtered input signal $[11,12]$. This assumption provides good results and is weaker than the hypothesis of the independence theory, which requires the statistical independence of time-lagged input data vectors.

Therefore, in what follows, we introduce the following approximation.

(A1) For every $i$ with $0 \leq i<K$ and for $m \in \mathbb{N}$, we assume $\mathbf{w}(m K+i)$ to be uncorrelated with $\mathbf{M}_{i}(m K+i)$ and with $\mathbf{q}_{\boldsymbol{\Sigma}, i}(m K+i)$.

In the appendix, we prove the following theorem that describes the transient behavior of the FX-PE-AP algorithm.

Theorem 1. Under the assumption (A1), the transient behavior of the FX-PE-AP algorithm with updating rule given by (15) is described by the state recursions

$$
\begin{aligned}
& E[\mathbf{w}(m K+i+K)]=\mathbf{M}_{i} E[\mathbf{w}(m K+i)]-\mathbf{m}_{i}, \\
& \mathbf{W}_{i}(m K+i+K)=\mathbf{G}_{i} \mathbf{W}_{i}(m K+i)+\mathbf{y}_{i}(m K+i),
\end{aligned}
$$


where

$$
\begin{aligned}
& \mathbf{M}_{i}=E\left[\mathbf{M}_{i}(n)\right], \\
& \mathbf{m}_{i}=E\left[\mathbf{m}_{i}(n)\right], \\
& \mathbf{G}_{i}=\left[\begin{array}{ccccc}
0 & 1 & 0 & \cdots & 0 \\
0 & 0 & 1 & \cdots & 0 \\
\vdots & \vdots & \vdots & \ddots & \vdots \\
0 & 0 & 0 & \cdots & 1 \\
-p_{0, i} & -p_{1, i} & -p_{2, i} & \cdots & -p_{M^{2}-1, i}
\end{array}\right] \\
& \mathbf{W}_{i}(n)=\left[\begin{array}{c}
E\left[\|\mathbf{w}(n)\|_{\mathrm{vec}^{-1}\{\boldsymbol{\sigma}\}}\right] \\
E\left[\|\mathbf{w}(n)\|_{\mathrm{vec}^{-1}\left\{\mathrm{~F}_{i} \boldsymbol{\sigma}\right\}}\right] \\
\vdots \\
E\left[\|\mathbf{w}(n)\|_{\mathrm{vec}^{-1}\left\{\mathbf{F}_{i}^{M^{2}-1} \boldsymbol{\sigma}\right\}}\right]
\end{array}\right], \\
& \mathbf{y}_{i}(n)=\left[\begin{array}{c}
\left(\mathbf{g}_{i}^{T}-2 E\left[\mathbf{w}^{T}(n)\right] \mathbf{Q}_{i}\right) \boldsymbol{\sigma} \\
\left(\mathbf{g}_{i}^{T}-2 E\left[\mathbf{w}^{T}(n)\right] \mathbf{Q}_{i}\right) \mathbf{F}_{i} \boldsymbol{\sigma} \\
\vdots \\
\left(\mathbf{g}_{i}^{T}-2 E\left[\mathbf{w}^{T}(n)\right] \mathbf{Q}_{i}\right) \mathbf{F}_{i}^{M^{2}-1} \boldsymbol{\sigma}
\end{array}\right],
\end{aligned}
$$

the $M^{2} \times M^{2}$ matrix $\mathbf{F}_{i}=E\left[\mathbf{M}_{i}^{T}(n) \otimes \mathbf{M}_{i}^{T}(n)\right]$, the $M \times M^{2}$ matrix $\mathbf{Q}_{i}=E\left[\mathbf{m}_{i}^{T}(n) \otimes \mathbf{M}_{i}^{T}(n)\right]$, the $M^{2} \times 1$ vector $\mathbf{g}_{i}=$ $\operatorname{vec}\left\{E\left[\mathbf{m}_{i}(n) \mathbf{m}_{i}^{T}(n)\right]\right\}$, the $p_{j, i}$ are the coefficients of the characteristic polynomial of $\mathbf{F}_{i}$, that is, $p_{i}(x)=x^{M^{2}}+p_{M^{2}-1, i} x^{M^{2}-1}+$ $\cdots+p_{1, i} x+p_{0, i}=\operatorname{det}\left(x \mathbf{I}-\mathbf{F}_{i}\right)$, and $\boldsymbol{\sigma}=\operatorname{vec}\{\boldsymbol{\Sigma}\}$.

Note that since the input signals are stationary, $\mathbf{M}_{i}, \mathbf{m}_{i}$, $\mathbf{G}_{i}, \mathbf{F}_{i}, \mathbf{Q}_{i}$, and $\mathbf{g}_{i}$, are time-independent. On the contrary, $\mathbf{y}_{i}(n)$ depends from the time sample $n$ through $E[\mathbf{w}(n)]$.

According to Theorem 1, for every index $i$ the transient behavior of the FX-PE-AP algorithm is described by the cascade of two linear systems, with system matrices $\mathbf{M}_{i}$ and $\mathbf{G}_{i}$, respectively. The stability in the mean sense and in the mean-square sense can be deduced by the stability properties of these two linear systems. Indeed, the FX-PE-AP algorithm will converge in the mean for any step-size $\mu$ such that for every $i,\left|\lambda_{\max }\left(\mathbf{M}_{i}\right)\right|<1$. The algorithm will converge in the mean-square sense if, in addition, for every $i$ it is $\left|\lambda_{\max }\left(\mathbf{F}_{i}\right)\right|<1$.

It should be noted that the matrices $\mathbf{M}_{i}$ and $\mathbf{F}_{i}$ are matrix polynomials in $\mu$ with degrees $K$ and $2 K$, respectively. Therefore, with the mild hypotheses of Theorem 1, an upper bound on the step-size that guarantees the mean and mean-square stabilities of the algorithm cannot be trivially determined. Nevertheless, the result of Theorem 1 could be used together with other more restrictive assumptions, for example on the statistics of the input signals, for deriving further descriptions of the transient behavior of the FX-PE-AP algorithm.
It should also be noted that the matrices $\mathbf{M}_{i}$ and $\mathbf{F}_{i}$ are nonsymmetric for both perfect and imperfect secondary path estimates. Thus, the algorithm could originate an oscillatory convergence behavior.

\subsection{Steady-state behavior}

We are here interested in the estimation of the mean-square error (MSE) and the mean-square deviation (MSD) at steady state. The adaptation rule of (15) provides different values of MSE and MSD for the different indexes $i$. Therefore, in what follows, we define

$$
\begin{aligned}
\operatorname{MSD}_{i} & =\lim _{m \rightarrow+\infty} E\left[\left\|\mathbf{w}(m K+i)-\mathbf{w}_{\infty, i}\right\|^{2}\right] \\
& =\lim _{m \rightarrow+\infty} E\left[\mathbf{w}^{T}(m K+i) \mathbf{w}(m K+i)\right]-\left\|\mathbf{w}_{\infty, i}\right\|^{2}, \\
\mathrm{MSE}_{i} & =\lim _{m \rightarrow+\infty} E\left[\sum_{k=1}^{K} e_{k}^{2}(m K+i)\right] .
\end{aligned}
$$

Note that the definition of the MSD in (27) refers to the asymptotic solution $\mathbf{w}_{\infty, i}$ instead of the mean-square solution $\mathbf{w}_{o}$ as in $[11,12,20]$. We adopt the definition in (27) because when $\mu$ tends to zero, also the MSD in (27) converges to zero, that is, $\lim _{\mu \rightarrow 0} \mathrm{MSD}_{i}=0$ for all $i$.

Similar to [4], we make use of the following hypothesis:

(A2) We assume $\mathbf{w}(n)$ to be uncorrelated with $\sum_{k=1}^{K} \mathbf{u}_{k}(n) \times$ $\mathbf{u}_{k}^{T}(n)$ and with $\sum_{k=1}^{K} d_{k}(n) \mathbf{u}_{k}(n)$.

By exploiting the hypothesis in (A2), the MSE can be expressed as

$\mathrm{MSE}_{i}=S_{d}+2 \mathbf{R}_{u d}^{T} \mathbf{w}_{\infty, i}+\lim _{m \rightarrow+\infty} E\left[\mathbf{w}^{T}(m K+i) \mathbf{R}_{u u} \mathbf{w}(m K+i)\right]$,

where

$$
S_{d}=E\left[\sum_{k=1}^{K} d_{k}^{2}(n)\right],
$$

and $\mathbf{R}_{u u}$ and $\mathbf{R}_{u d}$ are defined in (20), respectively.

The computations in (27) and (29) require the evaluation of $\lim _{m \rightarrow+\infty} E\left[\|\mathbf{w}(m K+i)\|_{\boldsymbol{\Sigma}}\right]$, where $\boldsymbol{\Sigma}=\mathbf{I}$ in (27) and $\boldsymbol{\Sigma}=\mathbf{R}_{u u}$ in (29). This limit can be estimated with the same methodology of [12].

If we assume the convergence of the algorithm, when $m \rightarrow+\infty$, the recursion in (A.1) becomes

$$
\begin{aligned}
\lim _{m \rightarrow+\infty} E\left[\|\mathbf{w}(m K+i)\|_{\mathrm{vec}^{-1}\{\boldsymbol{\sigma}\}}^{2}\right] \\
\quad=\lim _{m \rightarrow+\infty} E\left[\|\mathbf{w}(m K+i)\|_{\mathrm{vec}^{-1}\left\{\mathrm{~F}_{i} \boldsymbol{\sigma}\right\}}^{2}\right]-2 \mathbf{w}_{\infty, i}^{T} \mathbf{Q}_{i} \boldsymbol{\sigma}+\mathbf{g}_{i}^{T} \boldsymbol{\sigma},
\end{aligned}
$$

which is equivalent to

$$
\lim _{m \rightarrow+\infty} E\left[\|\mathbf{w}(m K+i)\|_{\operatorname{vec}^{-1}\left\{\left(\mathbf{I}-\mathbf{F}_{i}\right) \boldsymbol{\sigma}\right\}}^{2}\right]=-2 \mathbf{w}_{\infty, i}^{T} \mathbf{Q}_{i} \boldsymbol{\sigma}+\mathbf{g}_{i}^{T} \boldsymbol{\sigma} .
$$


TABLE 2: First eight coefficients of the MMS solution $\left(\mathbf{w}_{o}\right)$ and of the asymptotic solutions of FX-PE-AP $\left(\mathbf{w}_{\infty, 0}, \mathbf{w}_{\infty, 1}\right)$ and of FX-AP algorithm $\left(\mathbf{w}_{\infty}\right)$ with the linear controller.

\begin{tabular}{r|r|r|r|r|r|r|r|r|r}
\hline & \multicolumn{3}{|c|}{$L=1$} & \multicolumn{1}{c|}{$L=2$} & \multicolumn{3}{c}{$L=3$} \\
\hline \multicolumn{1}{c|}{$\mathbf{w}_{0}$} & $\mathbf{w}_{\infty, 0}$ & $\mathbf{w}_{\infty, 1}$ & \multicolumn{1}{c|}{$\mathbf{w}_{\infty}$} & $\mathbf{w}_{\infty, 0}$ & $\mathbf{w}_{\infty, 1}$ & $\mathbf{w}_{\infty}$ & $\mathbf{w}_{\infty, 0}$ & $\mathbf{w}_{\infty, 1}$ & $\mathbf{w}_{\infty}$ \\
\hline 0.808 & 0.868 & 0.886 & 0.847 & 0.735 & 0.746 & 0.787 & 0.799 & 0.796 & 0.818 \\
-0.692 & -0.749 & -0.769 & -0.732 & -0.620 & -0.604 & -0.679 & -0.755 & -0.717 & -0.738 \\
0.352 & 0.387 & 0.406 & 0.376 & 0.306 & 0.281 & 0.344 & 0.423 & 0.390 & 0.390 \\
-0.232 & -0.256 & -0.272 & -0.247 & -0.184 & -0.167 & -0.219 & -0.276 & -0.260 & -0.260 \\
0.154 & 0.159 & 0.168 & 0.158 & 0.136 & 0.112 & 0.154 & 0.201 & 0.181 & 0.183 \\
-0.086 & -0.083 & -0.093 & -0.082 & -0.060 & -0.052 & -0.075 & -0.099 & -0.088 & -0.093 \\
0.071 & 0.049 & 0.052 & 0.052 & 0.055 & 0.043 & 0.053 & 0.076 & 0.060 & 0.057 \\
-0.007 & -0.008 & -0.008 & -0.007 & -0.008 & 0.006 & -0.005 & -0.015 & 0.000 & -0.007 \\
\hline
\end{tabular}

To estimate the MSE, we have to choose $\boldsymbol{\sigma}$ such that (I $\left.\mathbf{F}_{i}\right) \boldsymbol{\sigma}=\operatorname{vec}\left\{\mathbf{R}_{u u}\right\}$, that is, $\boldsymbol{\sigma}=\left(\mathbf{I}-\mathbf{F}_{i}\right)^{-1} \operatorname{vec}\left\{\mathbf{R}_{u u}\right\}$. Therefore, the MSE can be evaluated as in

$\mathrm{MSE}_{i}=S_{d}+2 \mathbf{R}_{u d}^{T} \mathbf{w}_{\infty, i}+\left(\mathbf{g}_{i}^{T}-2 \mathbf{w}_{\infty, i}^{T} \mathbf{Q}_{i}\right)\left(\mathbf{I}-\mathbf{F}_{i}\right)^{-1} \operatorname{vec}\left\{\mathbf{R}_{u u}\right\}$.

To estimate the MSD, we have to choose $\boldsymbol{\sigma}$ such that (I $\left.\mathbf{F}_{i}\right) \boldsymbol{\sigma}=\operatorname{vec}\{\mathbf{I}\}$, that is, $\boldsymbol{\sigma}=\left(\mathbf{I}-\mathbf{F}_{i}\right)^{-1} \operatorname{vec}\{\mathbf{I}\}$. Thus, the MSD can be evaluated as in

$$
\operatorname{MSD}_{i}=\left(\mathbf{g}_{i}^{T}-2 \mathbf{w}_{\infty_{i}}^{T} \mathbf{Q}_{i}\right)\left(\mathbf{I}-\mathbf{F}_{i}\right)^{-1} \operatorname{vec}\{\mathbf{I}\}-\left\|\mathbf{w}_{\infty, i}\right\|^{2} .
$$

\section{EXPERIMENTAL RESULTS}

In this section, we provide a few experimental results that compare theoretically predicted values with values obtained from simulations.

We first considered a multichannel active noise controller with $I=1, J=2, K=2$. The transfer functions of the primary paths are given by

$$
\begin{aligned}
& p_{1,1}(z)=1.0 z^{-2}-0.3 z^{-3}+0.2 z^{-4}, \\
& p_{2,1}(z)=1.0 z^{-2}-0.2 z^{-3}+0.1 z^{-4},
\end{aligned}
$$

and the transfer functions of the secondary paths are

$$
\begin{aligned}
& s_{1,1}(z)=2.0 z^{-1}-0.5 z^{-2}+0.1 z^{-3}, \\
& s_{1,2}(z)=2.0 z^{-1}-0.3 z^{-2}-0.1 z^{-3}, \\
& s_{2,1}(z)=1.0 z^{-1}-0.7 z^{-2}-0.2 z^{-3}, \\
& s_{2,2}(z)=1.0 z^{-1}-0.2 z^{-2}+0.2 z^{-3} .
\end{aligned}
$$

For simplicity, we provide results only for a perfect estimate of the secondary paths, that is, we consider $\widetilde{s}_{i, j}(z)=s_{i, j}(z)$. The input signal is the normalized logistic noise, which has been generated by scaling the signal $\xi(n)$ obtained from the logistic recursion $\xi(n+1)=\lambda \xi(n)(1-\xi(n))$, with $\lambda=4$ and $\xi(0)=0.9$, and by adding a white Gaussian noise to get a $30 \mathrm{~dB}$ signal-to-noise ratio. It has been proven for singlechannel active noise controllers that in presence of a nonminimum phase secondary path, the controller acts as a predictor of the reference signal and that a nonlinear controller can better estimate a non-Gaussian noise process [15, 21]. In the case of our multichannel active noise controller, the exact solution of the multichannel ANC problem requires the inversion of the $2 \times 2$ matrix $\boldsymbol{S}$ formed with the transfer functions $s_{k, j}$. The inverse matrix $\mathbf{S}^{-1}$ is formed by IIR transfer functions whose poles are given by the roots of the determinant of $\mathbf{S}$. It is easy to verify that in our example, there is a root outside the unit circle. Thus, also in our case the controller acts as a predictor of the input signal and a nonlinear controller can better estimate the logistic noise. Therefore, in what follows, we provide results for (1) the two-channel linear controller with memory length $N=8$ and (2) the two-channel nonlinear controller with memory length $N=4$ whose input data vector is given in (4), with the constant $a$ set to 1 . Note that despite the two controllers have different memory lengths, they have the same total number of coefficients, that is, $M=16$. In all the experiments, a zero mean, white Gaussian noise, uncorrelated between the microphones, has been added to the error microphone signals $d_{k}(n)$ to get a $40 \mathrm{~dB}$ signal-to-noise ratio and the parameter $\delta$ was set to 0.001 .

Tables 2 and 3 provide with three-digits precision the first eight coefficients of the MMS solution, $\mathbf{w}_{o}$, and of the asymptotic solutions of the FX-PE-AP algorithm at even samples, $\mathbf{w}_{\infty, 0}$, and odd samples, $\mathbf{w}_{\infty, 1}$, and of the approximate FX-AP algorithm of (6), $\mathbf{w}_{\infty}$, for $\mu=1.0$ and for the AP orders $L=1$, 2 , and 3. Table 2 refers to the linear controller and Table 3 to the nonlinear controller, respectively. From Tables 2 and 3, it is evident that the asymptotic vector varies with the AP order and that the asymptotic solutions $\mathbf{w}_{\infty, 0}, \mathbf{w}_{\infty, 1}$, and $\mathbf{w}_{\infty}$ are different. However, we must point out that their difference reduces with the step-size, and for smaller step-sizes it can be difficulty appreciated.

Figure 3 diagrams the steady-state MSE, estimated with (33) or obtained from simulations with time averages over ten million samples, versus step-size $\mu$ and for AP orders $L=1,2$, and 3. Similarly, Figure 4 diagrams the steady-state MSD, estimated with (34) or obtained from simulations with time averages over ten million samples. From Figures 3 and 4 , we see that the expressions in (33) and in (34) provide accurate estimates of the steady-state MSE and of the steady-state 
TABLE 3: First eight coefficients of the MMS solution $\left(\mathbf{w}_{o}\right)$ and of the asymptotic solutions of FX-PE-AP $\left(\mathbf{w}_{\infty, 0}, \mathbf{w}_{\infty, 1}\right)$ and of FX-AP algorithm $\left(\mathbf{w}_{\infty}\right)$ with the nonlinear controller.

\begin{tabular}{|c|c|c|c|c|c|c|c|c|c|}
\hline \multirow[b]{2}{*}{$\mathbf{w}_{o}$} & \multicolumn{3}{|c|}{$L=1$} & \multicolumn{3}{|c|}{$L=2$} & \multicolumn{3}{|c|}{$L=3$} \\
\hline & $\mathbf{w}_{\infty, 0}$ & $\mathbf{w}_{\infty, 1}$ & $\mathbf{w}_{\infty}$ & $\mathbf{w}_{\infty, 0}$ & $\mathbf{w}_{\infty, 1}$ & $\mathbf{w}_{\infty}$ & $\mathbf{w}_{\infty, 0}$ & $\mathbf{w}_{\infty, 1}$ & $\mathbf{w}_{\infty}$ \\
\hline 0.566 & 0.699 & 0.673 & 0.644 & 0.445 & 0.481 & 0.560 & 0.600 & 0.602 & 0.625 \\
\hline-0.352 & -0.448 & -0.459 & -0.415 & -0.259 & -0.259 & -0.333 & -0.394 & -0.354 & -0.370 \\
\hline 0.172 & 0.163 & 0.169 & 0.168 & 0.216 & 0.175 & 0.173 & 0.194 & 0.152 & 0.141 \\
\hline 0.042 & -0.005 & 0.021 & 0.022 & 0.029 & 0.048 & 0.039 & 0.030 & 0.044 & 0.039 \\
\hline-0.877 & -0.755 & -0.745 & -0.816 & -1.021 & -0.991 & -0.884 & -0.801 & -0.809 & -0.736 \\
\hline 0.755 & 0.865 & 0.792 & 0.821 & 0.659 & 0.754 & 0.731 & 0.636 & 0.711 & 0.682 \\
\hline-0.230 & -0.434 & -0.406 & -0.367 & 0.005 & -0.122 & -0.201 & -0.177 & -0.234 & -0.247 \\
\hline 0.268 & 0.269 & 0.307 & 0.292 & 0.276 & 0.255 & 0.266 & 0.269 & 0.229 & 0.220 \\
\hline
\end{tabular}

MSD, respectively, when $L=2$ and $L=3$. The estimation errors can be both positive or negative depending on the AP order, the step-size, and the odd or even sample times. On the contrary, for the AP order $L=1$, the estimations are inaccurate. The large estimation errors for $L=1$ are due to the bad conditioning of the matrices $\mathbf{M}_{i}-\mathbf{I}$ that takes to a poor estimate of the asymptotic solution. For larger AP orders, the data reuse property of the AP algorithm takes to more regular matrices $\mathbf{M}_{i}$. Indeed, Table 4 compares the condition number, that is, the ratio between the magnitude of the largest and the smallest of the eigenvalues of the matrix $\mathbf{M}_{i}$ - I of the nonlinear controller at even-time indexes for the AP orders $L=1,2$, and 3 and for different values of the step-size.

Figures 5 and 6 diagram the ensemble averages, estimated over 100 runs of the FX-PE-AP and the FX-AP algorithms with step-size equal to 0.032 , of the mean value of the residual power of the error computed on 100 successive samples for the nonlinear and the linear controllers, respectively. In the figures, the asymptotic values (dashed lines) of the residual power of the errors are also shown. From Figures 5 and 6 , it is evident that the nonlinear controller outperforms the linear one in terms of residual error. Nevertheless, it must be observed that the nonlinear controller reaches the steadystate condition in a slightly longer time than the linear controller. This behavior could also be predicted by the maximum eigenvalues of the matrices $\mathbf{M}_{i}$ and $\mathbf{F}_{i}$, which are reported in Table 5 . Since the step-size $\mu$ assumes a small value $(\mu=0.032)$, in the table we have the same maximum eigenvalue for $\mathbf{M}_{0}$ and $\mathbf{M}_{1}$ and for $\mathbf{F}_{0}$ and $\mathbf{F}_{1}$. Moreover, as already observed for the filtered- $x$ PE LMS algorithm [2], from Figures 5 and 6 it is apparent that for this step-size, the FX-PE$\mathrm{AP}$ algorithm has a convergence speed that is half (i.e., $1 / K$ ) of the approximate FX-AP algorithm. In fact, the diagrams on the left and the right of the figures can be overlapped but the time scale of the FX-PE-AP algorithm is the double of the FX-AP algorithm. The same observation applies also when a larger number of microphones are considered. For example, Figures 7 and 8 plot the ensemble averages, estimated over 100 runs of the FX-PE-AP and the FX-AP algorithm with step-size equal to 0.032 , of the mean value of the residual power of the error computed on 100 successive samples for the nonlinear controller with $I=1, J=2, K=3$, and with $I=1, J=2, K=4$, respectively. In the case $I=1, J=2$, $K=3$, the transfer functions of the primary paths, $p_{1,1}(z)$ and $p_{2,1}(z)$, and of the secondary paths, $s_{1,1}(z), s_{1,2}(z), s_{2,1}(z)$, and $s_{2,2}(z)$, are given by (35)-(36), while the other primary and secondary paths are given by

$$
\begin{aligned}
& p_{3,1}(z)=1.0 z^{-2}-0.3 z^{-3}+0.1 z^{-4} \\
& s_{3,1}(z)=1.6 z^{-1}-0.6 z^{-2}+0.1 z^{-3} \\
& s_{3,2}(z)=1.6 z^{-1}-0.2 z^{-2}-0.1 z^{-3}
\end{aligned}
$$

In the case $I=1, J=2, K=4$, the transfer functions of the primary paths, $p_{1,1}(z), p_{2,1}(z)$, and $p_{3,1}(z)$, and of the secondary paths, $s_{1,1}(z), s_{1,2}(z), s_{2,1}(z), s_{2,2}(z), s_{3,1}(z)$, and $s_{3,2}(z)$, are given by (35)-(37), and the other primary and secondary paths are given by

$$
\begin{gathered}
p_{4,1}(z)=1.0 z^{-2}-0.2 z^{-3}+0.2 z^{-4} \\
s_{4,1}(z)=1.3 z^{-1}-0.5 z^{-2}-0.2 z^{-3} \\
s_{4,2}(z)=1.3 z^{-1}-0.4 z^{-2}+0.2 z^{-3}
\end{gathered}
$$

All the other experimental conditions are the same of the case $I=1, J=2, K=2$. Figures 7 and 8 confirm again that for $\mu=0.032$, the FX-PE-AP algorithm has a convergence speed that is reduced by a factor $K$ with respect to the approximate FX-AP algorithm. Nevertheless, we must point out that for larger values of the step-size, the reduction of convergence speed of the FX-PE-AP algorithm can be even larger than a factor $K$.

We have also performed the same simulations by reducing the SNR at the error microphones to 30,20 , and $10 \mathrm{~dB}$ and we have obtained similar convergence behaviors. The main difference, apart from the increase in the residual error, has been that the lowest is the SNR at the error microphones, the lowest is the improvement in the convergence speed obtained by increasing the affine projection order. 

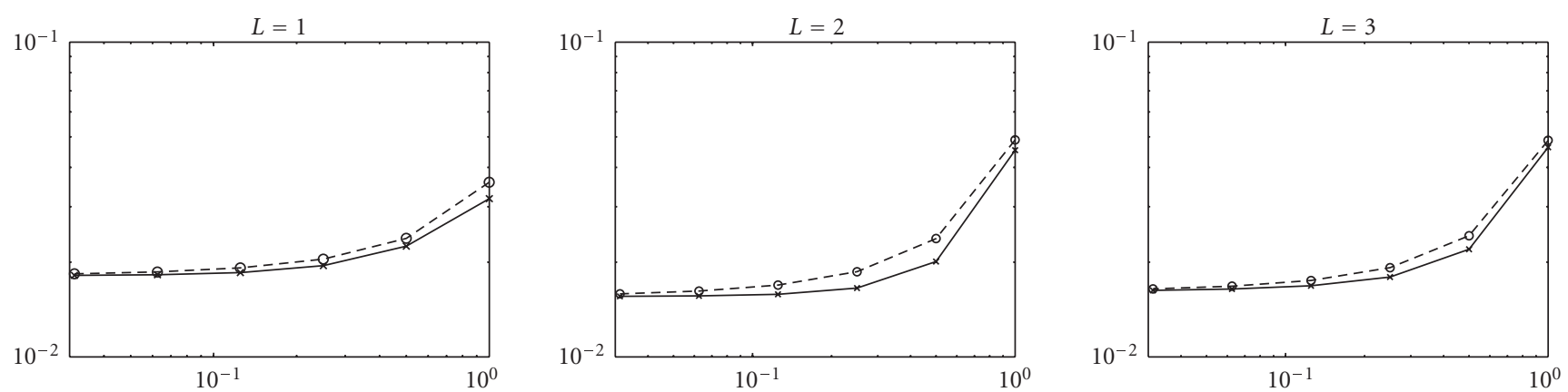

(a)
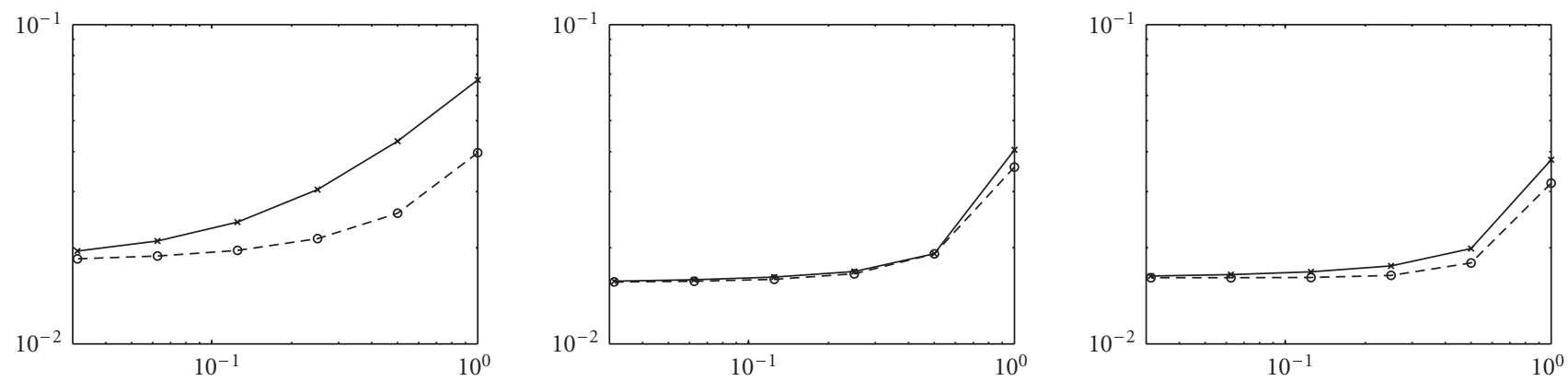

(b)
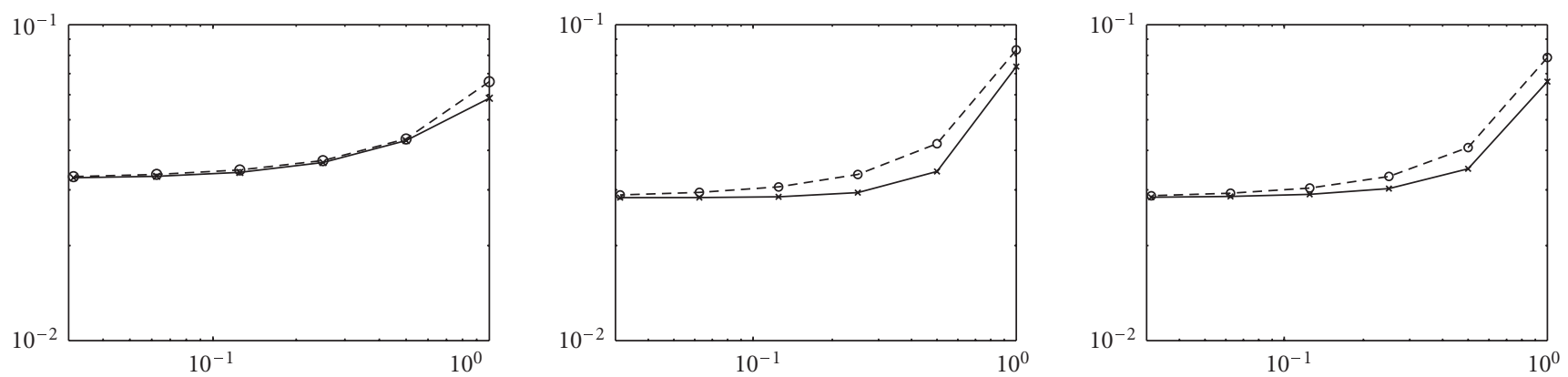

(c)
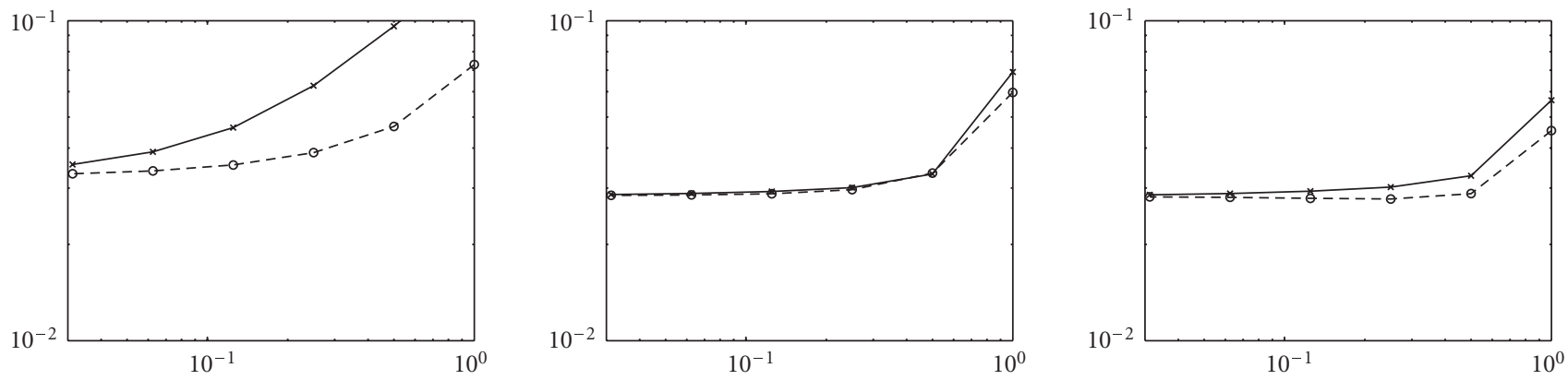

(d)

FIgure 3: Theoretical (- -) and simulation values (-) of steady-state MSE versus step-size of the FX-PE-AP algorithm (a) at even samples with a nonlinear controller, (b) at odd samples with a nonlinear controller, (c) at even samples with a linear controller, (d) at odd samples with a linear controller, for $L=1,2$, and 3 . 

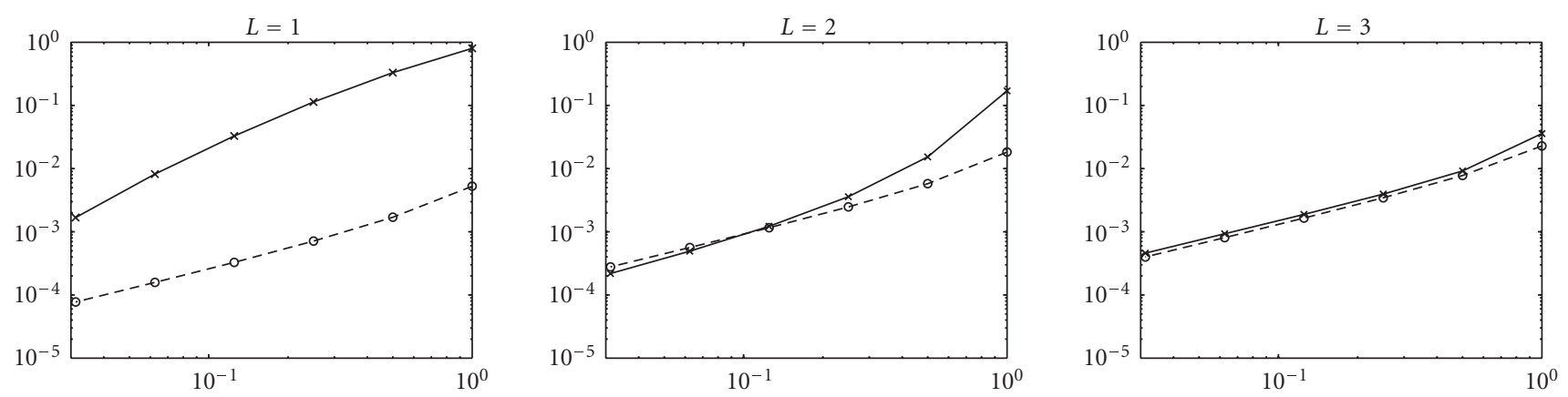

(a)
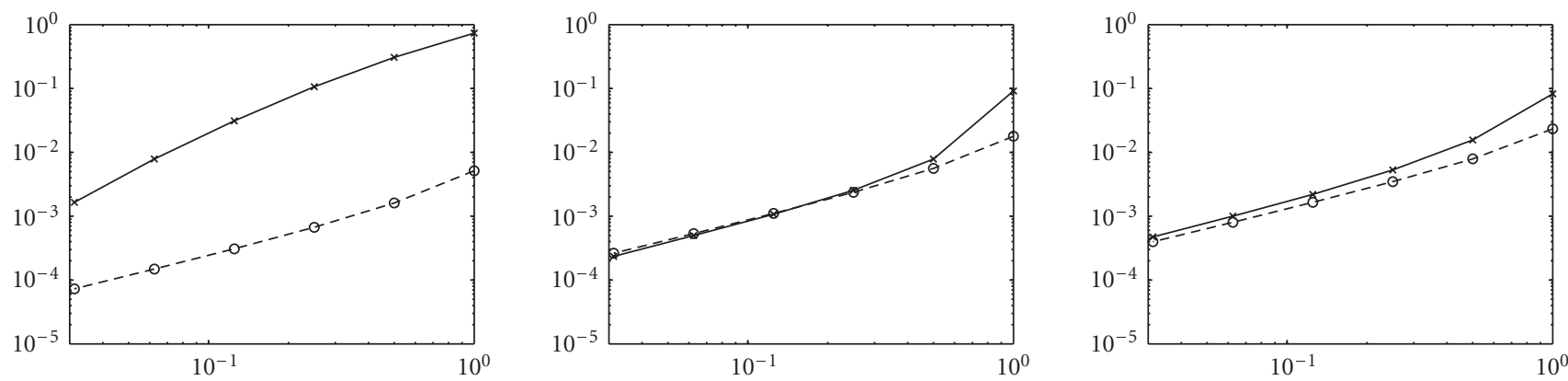

(b)
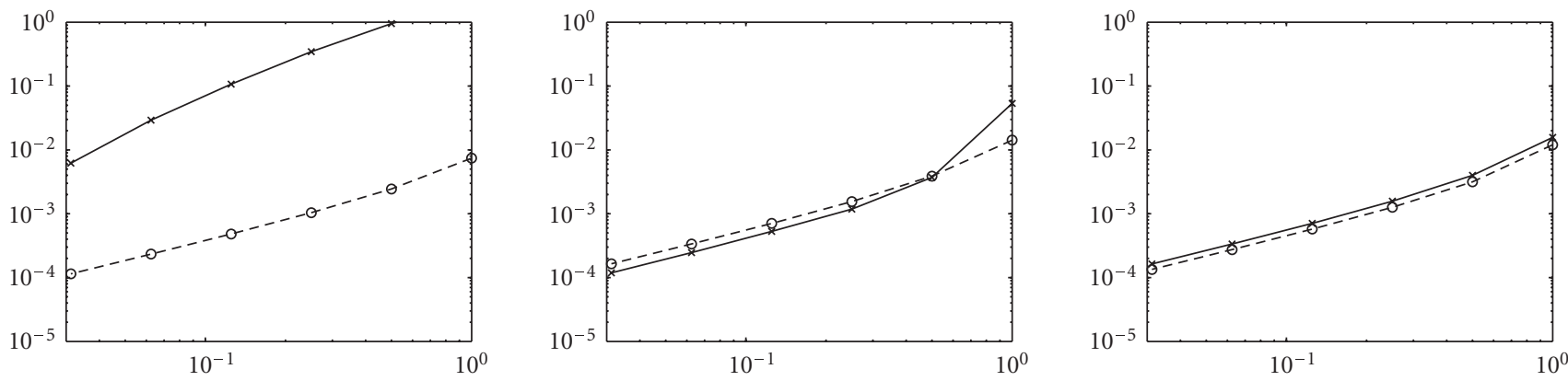

(c)
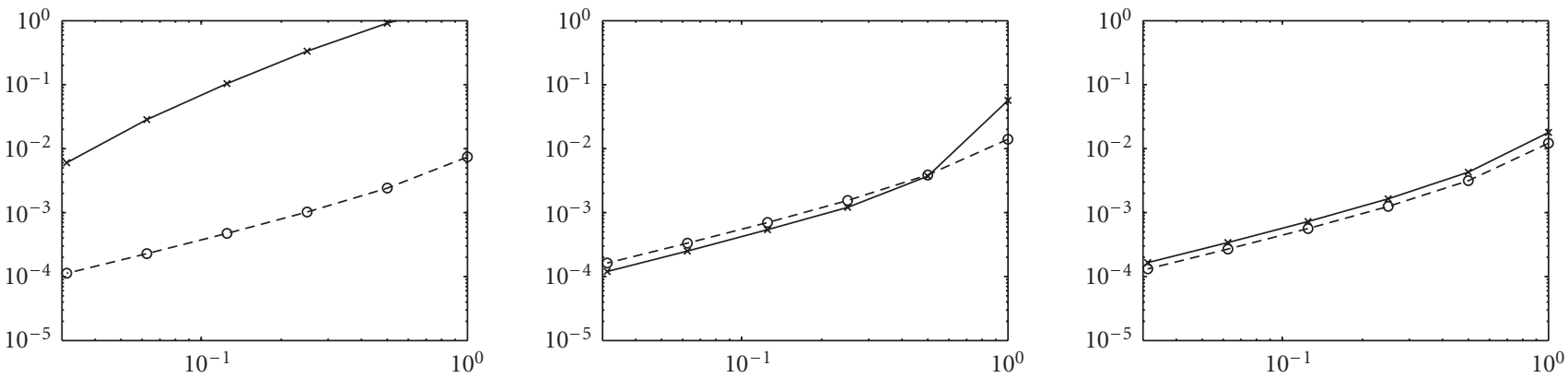

(d)

FIgUre 4: Theoretical (- -) and simulation values (-) of steady-state MSD versus step-size of the FX-PE-AP algorithm (a) at even samples with a nonlinear controller, (b) at odd samples with a nonlinear controller, (c) at even samples with a linear controller, (d) at odd samples with a linear controller, for $L=1,2$, and 3 . 


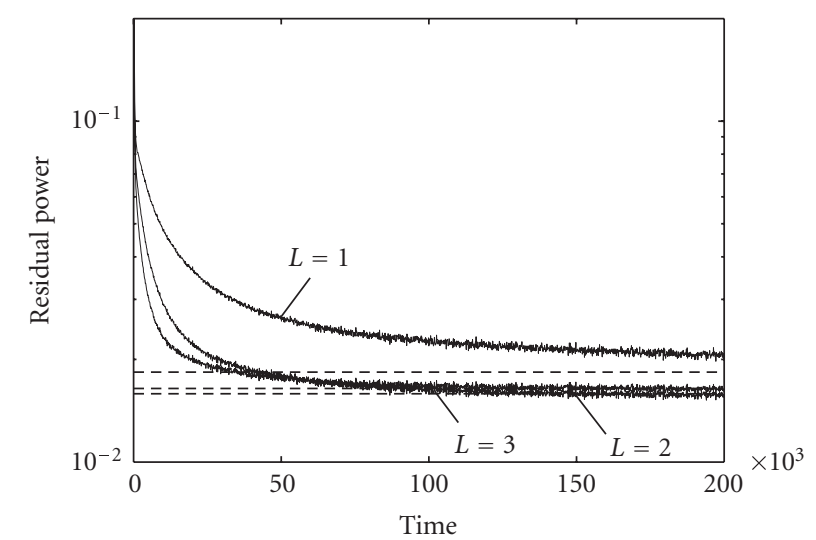

(a)

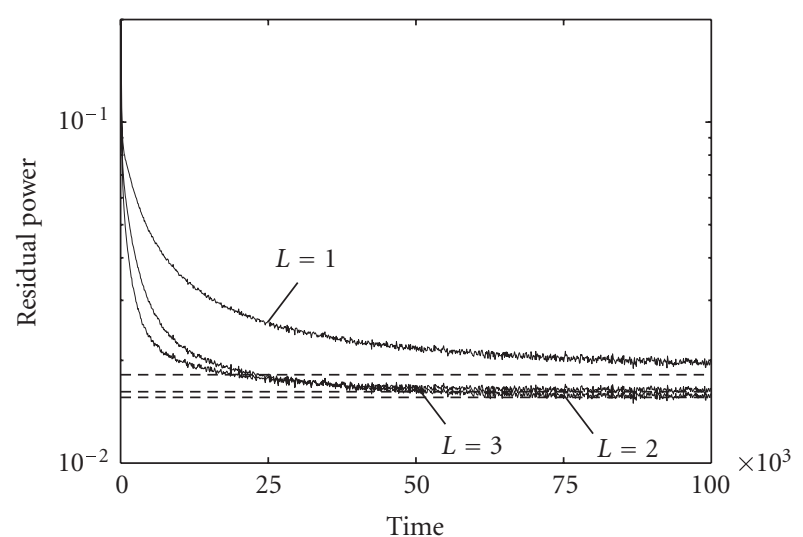

(b)

FIGURE 5: Evolution of residual power of the error of (a) the FX-PE-AP algorithm and (b) FX-AP algorithm with a nonlinear controller and $I=1, J=2, K=2$. The dashed lines diagram the asymptotic values of the residual power.

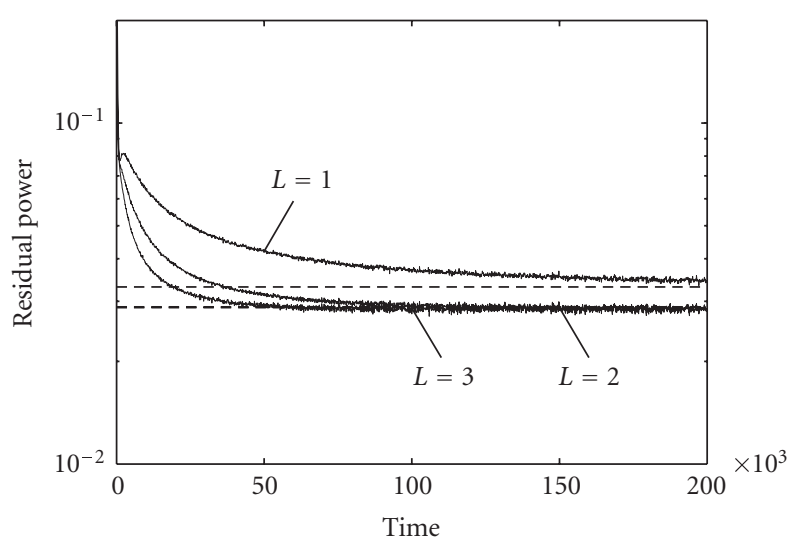

(a)

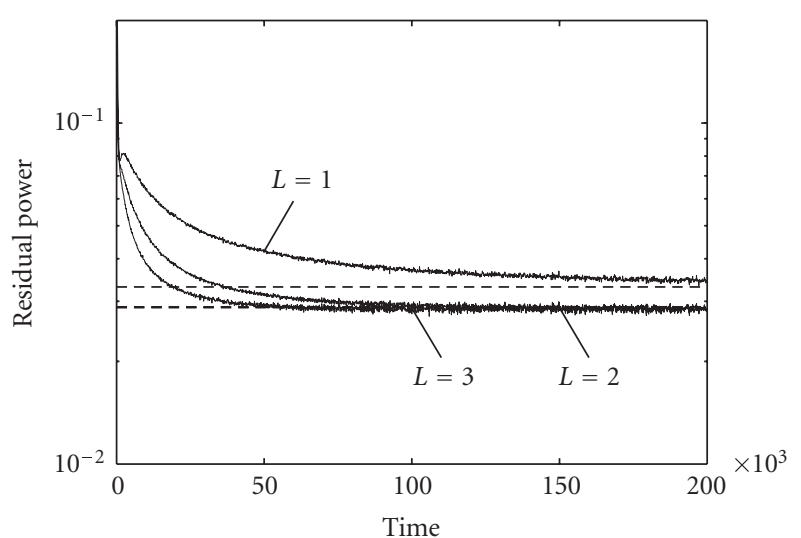

(b)

FIgURE 6: Evolution of residual power of the error of (a) the FX-PE-AP algorithm and (b) FX-AP algorithm with a linear controller and $I=1, J=2, K=2$. The dashed lines diagram the asymptotic values of the residual power.

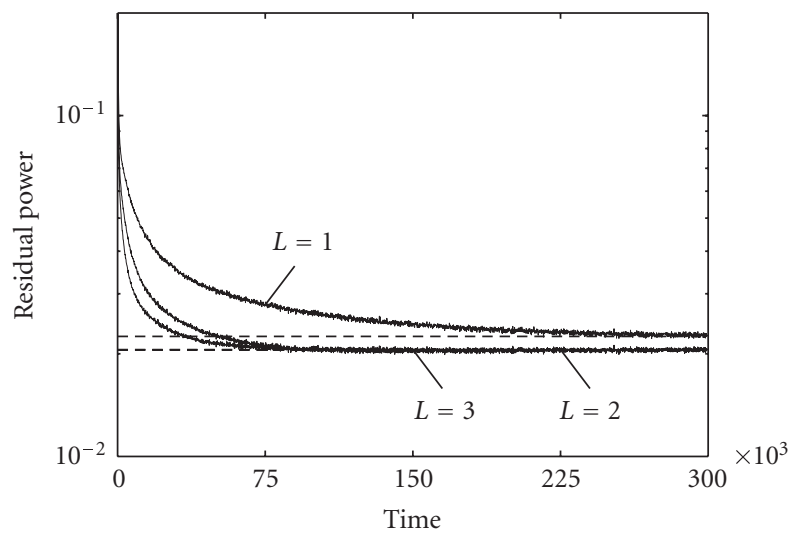

(a)

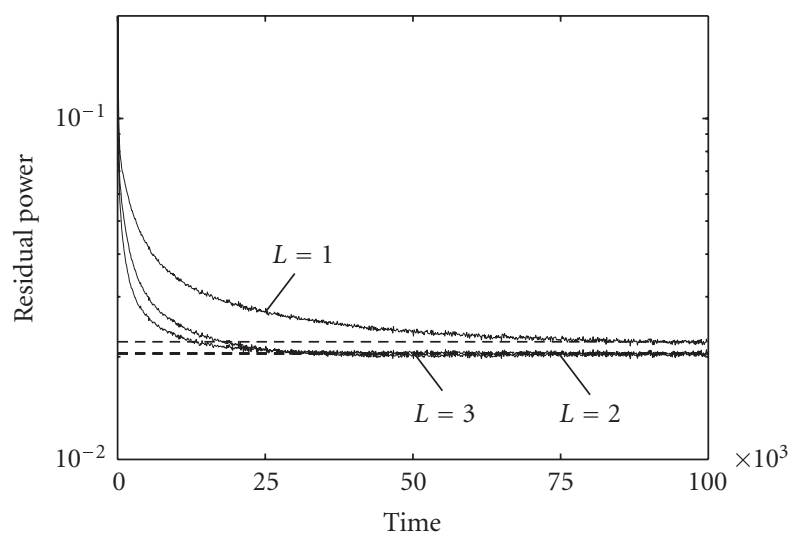

(b)

FIgURe 7: Evolution of residual power of the error of (a) the FX-PE-AP algorithm and (b) FX-AP algorithm with a nonlinear controller and $I=1, J=2, K=3$. The dashed lines diagram the asymptotic values of the residual power. 


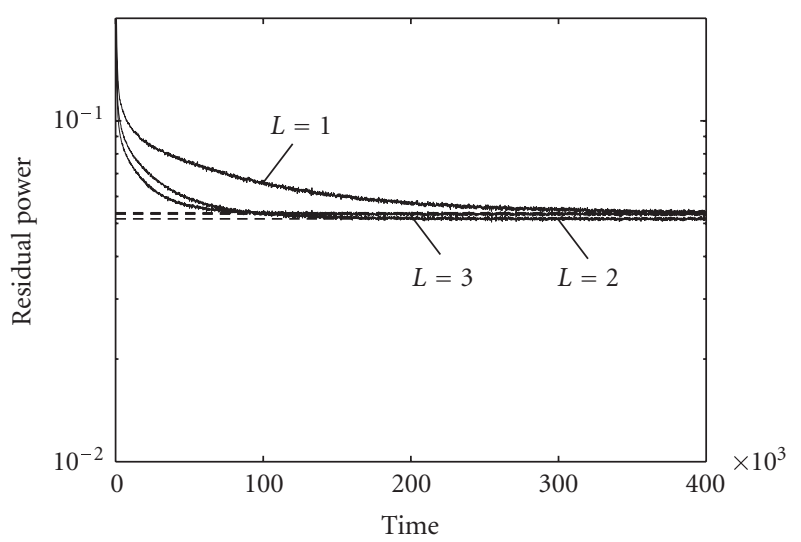

(a)

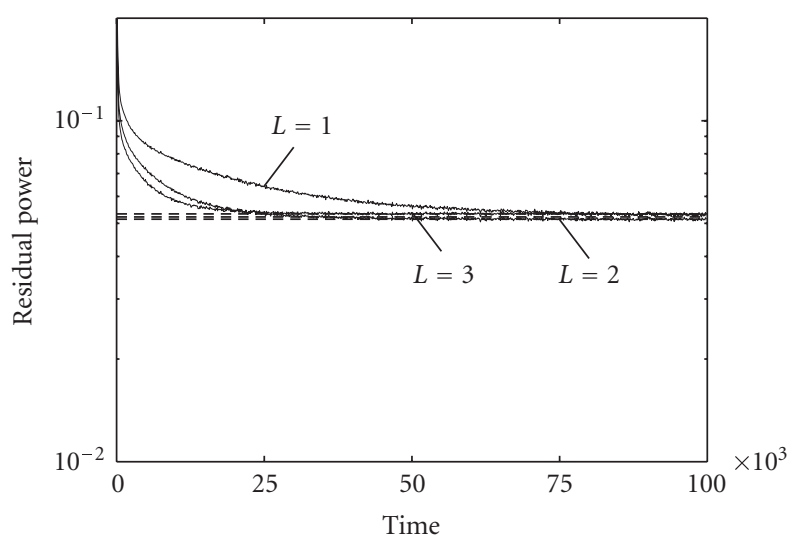

(b)

FIGURE 8: Evolution of residual power of the error of (a) the FX-PE-AP algorithm and (b) FX-AP algorithm with a nonlinear controller and $I=1, J=2, K=4$. The dashed lines diagram the asymptotic values of the residual power.

TABle 4: Condition number of the matrix $\mathbf{M}_{0}-\mathbf{I}$ for different stepsizes and for the AP orders $L=1,2$, and 3 with the nonlinear controller.

\begin{tabular}{l|c|c|c}
\hline$L$ & $\mu=1.0$ & $\mu=0.25$ & $\mu=0.0625$ \\
\hline$L=1$ & 33379 & 36299 & 36965 \\
$L=2$ & 6428 & 9711 & 10575 \\
$L=3$ & 2004 & 3290 & 3623 \\
\hline
\end{tabular}

TABle 5: Maximum eigenvalues of the matrices $\mathbf{M}_{i}$ and $\mathbf{F}_{i}$ for the AP orders $L=1,2$, and 3 with the linear and the nonlinear controllers.

\begin{tabular}{l|c|c|c|c}
\hline Controllers & & $L=1$ & $L=2$ & $L=3$ \\
\hline \multirow{2}{*}{ Nonlinear } & $\lambda_{\max }\left(\mathbf{M}_{i}\right)$ & 0.999999 & 0.999996 & 0.999987 \\
& $\lambda_{\max }\left(\mathbf{F}_{i}\right)$ & 0.999998 & 0.999992 & 0.999974 \\
\hline \multirow{2}{*}{ Linear } & $\lambda_{\max }\left(\mathbf{M}_{i}\right)$ & 0.999991 & 0.999972 & 0.999957 \\
& $\lambda_{\max }\left(\mathbf{F}_{i}\right)$ & 0.999981 & 0.999944 & 0.999914 \\
\hline
\end{tabular}

\section{CONCLUSION}

In this paper, we have provided an analysis of the transient and the steady-state behavior of the FX-PE-AP algorithm. We have shown that the algorithm in presence of stationary input signals converges to a cyclostationary process, that is, the asymptotic value of the coefficient vector, the meansquare error and the mean-square deviation tend to periodic functions of the sample time. We have shown that the asymptotic coefficient vector of the FX-PE-AP algorithm differs from the minimum-mean-square solution of the ANC problem and from the asymptotic solution of the AP algorithm from which the FX-PE-AP algorithm was derived. We have proved that the transient behavior of the algorithm can be studied by the cascade of two linear systems. By studying the system matrices of these two linear systems, we can predict the stability and the convergence speed of the algorithm. Expressions have been derived for the steady-state MSE and MSD of the FX-PE-AP algorithm. Eventually, we have com- pared the FX-PE-AP with the approximate FX-AP algorithm introduced in [4]. Compared with the approximate FX-AP algorithm, the FX-PE-AP algorithm is capable of reducing the adaptation complexity with a factor $K$. Nevertheless, also the convergence speed of the algorithm reduces of the same value.

\section{APPENDIX}

\section{PROOF OF THEOREM 1}

If we apply the expectation operator to both sides of (23), and if we take into account the hypothesis in (A1), we can derive the result of

$$
\begin{aligned}
E\left[\|\mathbf{w}(m K+i+K)\|_{\Sigma}^{2}\right] \\
=E\left[\|\mathbf{w}(m K+i)\|_{\mathbf{\Sigma}_{i}^{\prime}}^{2}\right]-2 E\left[\mathbf{w}^{T}(m K+i)\right] E\left[\mathbf{q}_{\boldsymbol{\Sigma}, i}(m K+i)\right] \\
\quad+E\left[\mathbf{m}_{i}^{T}(m K+i) \mathbf{\Sigma} \mathbf{m}_{i}(m K+i)\right],
\end{aligned}
$$

where

$$
\boldsymbol{\Sigma}^{\prime}{ }_{i}=E\left[\mathbf{M}_{i}^{T}(n) \boldsymbol{\Sigma} \mathbf{M}_{i}(n)\right] .
$$

Moreover, under the same hypothesis (A1), the evolution of the mean of the coefficient vector from (15) is described by

$$
\begin{aligned}
E[\mathbf{w}(m K+i+K)]= & E\left[\mathbf{M}_{i}(m K+i)\right] E[\mathbf{w}(m K+i)] \\
& -E\left[\mathbf{m}_{i}(m K+i)\right] .
\end{aligned}
$$

We manipulate (A.1), (A.2), and (A.3) by taking advantage of the properties of the vector operator vec $\{\cdot\}$ and of the Kronecker product, $\otimes$. We introduce the vectors $\boldsymbol{\sigma}=\operatorname{vec}\{\boldsymbol{\Sigma}\}$ and $\boldsymbol{\sigma}^{\prime}=\operatorname{vec}\left\{\boldsymbol{\Sigma}^{\prime}\right\}$. Since for any matrices $\mathbf{A}, \mathbf{B}$, and $\mathbf{C}$, it is

$$
\operatorname{vec}\{\mathbf{A B C}\}=\left(\mathbf{C}^{T} \otimes \mathbf{A}\right) \operatorname{vec}\{\mathbf{B}\},
$$

we have from (A.2) that

$$
\sigma^{\prime}=\mathrm{F}_{i} \sigma
$$


where $\mathbf{F}_{i}$ is the $M^{2} \times M^{2}$ matrix defined by

$$
\mathbf{F}_{i}=E\left[\mathbf{M}_{i}^{T}(n) \otimes \mathbf{M}_{i}^{T}(n)\right] .
$$

The product $E\left[\mathbf{q}_{\Sigma, i}^{T}(n)\right] E[\mathbf{w}(n)]$ can be evaluated as in

$$
\begin{aligned}
E\left[\mathbf{w}^{T}(n)\right] E\left[\mathbf{q}_{\boldsymbol{\Sigma}, i}(n)\right] & =\operatorname{Tr}\left\{E\left[\mathbf{w}^{T}(n)\right] E\left[\mathbf{q}_{\boldsymbol{\Sigma}, i}(n)\right]\right\} \\
& =E\left[\mathbf{w}^{T}(n)\right] \operatorname{vec}\left\{E\left[\mathbf{q}_{\boldsymbol{\Sigma}, i}(n)\right]\right\},
\end{aligned}
$$

with

$$
\begin{aligned}
\operatorname{vec}\left\{E\left[\mathbf{q}_{\boldsymbol{\Sigma}, i}(n)\right]\right\} & =\operatorname{vec}\left\{E\left[\mathbf{M}_{i}^{T}(n) \mathbf{\Sigma} \mathbf{m}_{i}(n)\right]\right\} \\
& =E\left[\mathbf{m}_{i}^{T}(n) \otimes \mathbf{M}_{i}^{T}(n)\right] \boldsymbol{\sigma}=\mathbf{Q}_{i}, \boldsymbol{\sigma},
\end{aligned}
$$

and the $M \times M^{2}$ matrix $\mathbf{Q}_{i}$ is given by

$$
\mathbf{Q}_{i}=E\left[\mathbf{m}_{i}^{T}(n) \otimes \mathbf{M}_{i}^{T}(n)\right] .
$$

Moreover, the last term of (A.1) can be computed as in

$$
\operatorname{Tr}\left\{E\left[\mathbf{m}_{i}^{T}(n) \mathbf{\Sigma} \mathbf{m}_{i}(n)\right]\right\}=\mathbf{g}_{i}^{T} \boldsymbol{\sigma},
$$

where

$$
\mathbf{g}_{i}=\operatorname{vec}\left\{E\left[\mathbf{m}_{i}(n) \mathbf{m}_{i}^{T}(n)\right]\right\}
$$

Accordingly, introducing $\boldsymbol{\sigma}$ and $\boldsymbol{\sigma}^{\prime}$ instead of $\boldsymbol{\Sigma}$ and $\boldsymbol{\Sigma}^{\prime}$ and using the results of (A.5), (A.7), (A.8), and (A.10), the recursion in (A.1) can be rewritten as follows:

$$
\begin{aligned}
& E\left[\|\mathbf{w}(m K+i+K)\|_{\mathrm{vec}^{-1}\{\boldsymbol{\sigma}\}}^{2}\right] \\
& \quad=E\left[\|\mathbf{w}(m K+i)\|_{\mathrm{vec}^{-1}\left\{\mathbf{F}_{i} \boldsymbol{\sigma}\right\}}^{2}\right]-2 E\left[\mathbf{w}^{T}(m K+i)\right] \mathbf{Q}_{i} \boldsymbol{\sigma}+\mathbf{g}_{i}^{T} \boldsymbol{\sigma} .
\end{aligned}
$$

The recursion in (A.12) shows that in order to evaluate $E\left[\|\mathbf{w}(m K+i+K)\|_{\mathrm{vec}^{-1}\{\boldsymbol{\sigma}\}}^{2}\right]$, we need $E\left[\|\mathbf{w}(m K+i)\|_{\mathrm{vec}^{-1}\left\{\mathbf{F}_{i} \boldsymbol{\sigma}\right\}}^{2}\right]$. This quantity can be inferred from (A.12) by replacing $\sigma$ with $\mathbf{F}_{i} \boldsymbol{\sigma}$, obtaining the following relation:

$$
\begin{aligned}
E[\| \mathbf{w} & \left.(m K+i+K) \|_{\mathrm{vec}^{-1}\left\{\mathbf{F}_{i} \boldsymbol{\sigma}\right\}}^{2}\right] \\
= & E\left[\|\mathbf{w}(m K+i)\|_{\mathrm{vec}^{-1}\left\{\mathbf{F}_{i}^{2} \boldsymbol{\sigma}\right\}}^{2}\right] \\
& -2 E\left[\mathbf{w}^{T}(m K+i)\right] \mathbf{Q}_{i} \mathbf{F}_{i} \boldsymbol{\sigma}+\mathbf{g}_{i}^{T} \mathbf{F}_{i} \boldsymbol{\sigma} .
\end{aligned}
$$

This procedure is repeated until we obtain the following expression $[12,18,19]$ :

$$
\begin{aligned}
E[\| \mathbf{w}( & \left.m K+i+K) \|_{\mathrm{vec}^{-1}\left\{\mathbf{F}_{i}^{M^{2}-1} \boldsymbol{\sigma}\right\}}^{2}\right] \\
= & E\left[\|\mathbf{w}(m K+i)\|_{\mathrm{vec}^{-1}\left\{\mathbf{F}_{i}^{M^{2}} \boldsymbol{\sigma}\right\}}^{2}\right] \\
& -2 E\left[\mathbf{w}^{T}(m K+i)\right] \mathbf{Q}_{i} \mathbf{F}_{i}^{M^{2}-1} \boldsymbol{\sigma}+\mathbf{g}_{i}^{T} \mathbf{F}_{i}^{M^{2}-1} \boldsymbol{\sigma} .
\end{aligned}
$$

According to the Cayley-Hamilton theorem, the matrix $\mathbf{F}_{i}$ satisfies its own characteristic equation. Therefore, if we indicate with $p_{i}(x)$ the characteristic polynomial of $\mathbf{F}_{i}, p_{i}(x)=$ $\operatorname{det}\left(x \mathbf{I}-\mathbf{F}_{i}\right)$, for the Cayley-Hamilton theorem we have that
$p_{i}\left(\mathbf{F}_{i}\right)=0$. The characteristic polynomial $p_{i}(x)$ is an order $M^{2}$ polynomial that can be written as in

$$
p_{i}(x)=x^{M^{2}}+p_{M^{2}-1, i} x^{M^{2}-1}+\cdots+p_{0, i},
$$

where we indicate with $\left\{p_{j, i}\right\}$ the coefficients of the polynomial. Since $p_{i}\left(\mathbf{F}_{i}\right)=0$, we deduce that $[12,18,19]$

$$
E\left[\|\mathbf{w}(n)\|_{\mathrm{vec}^{-1}\left\{\mathbf{F}_{i}^{M^{2}} \boldsymbol{\sigma}\right\}}^{2}\right]=-\sum_{j=0}^{M^{2}-1} p_{j, i} E\left[\|\mathbf{w}(n)\|_{\mathrm{vec}^{-1}\left\{\mathbf{F}_{i}^{j} \boldsymbol{\sigma}\right\}}^{2}\right] .
$$

The results of (A.3), (A.12)-(A.14), and (A.16) prove Theorem 1 that describes the transient behavior of the FXPE-AP algorithms.

\section{ACKNOWLEDGMENT}

This work was supported by MIUR under Grant PRIN 2004092314.

\section{REFERENCES}

[1] P. A. Nelson and S. J. Elliott, Active Control of Sound, Academic Press, London, UK, 1995.

[2] S. C. Douglas, "Fast implementations of the filtered-X LMS and LMS algorithms for multichannel active noise control," IEEE Transactions on Speech and Audio Processing, vol. 7, no. 4, pp. 454-465, 1999.

[3] M. Bouchard, "Multichannel affine and fast affine projection algorithms for active noise control and acoustic equalization systems," IEEE Transactions on Speech and Audio Processing, vol. 11, no. 1, pp. 54-60, 2003.

[4] A. Carini and G. L. Sicuranza, "Transient and steady-state analysis of filtered- $x$ affine projection algorithms," IEEE Transactions on Signal Processing, vol. 54, no. 2, pp. 665-678, 2006.

[5] Y. Neuvo, C.-Y. Dong, and S. K. Mitra, "Interpolated finite impulse response filters," IEEE Transactions on Acoustics, Speech, and Signal Processing, vol. 32, no. 3, pp. 563-570, 1984.

[6] S. Werner and P. S. R. Diniz, "Set-membership affine projection algorithm," IEEE Signal Processing Letters, vol. 8, no. 8, pp. 231-235, 2001.

[7] S. C. Douglas, "Adaptive filters employing partial updates," IEEE Transactions on Circuits and Systems II: Analog and Digital Signal Processing, vol. 44, no. 3, pp. 209-216, 1997.

[8] K. Doğançay and O. Tanrikulu, "Adaptive filtering algorithms with selective partial updates," IEEE Transactions on Circuits and Systems II: Analog and Digital Signal Processing, vol. 48, no. 8, pp. 762-769, 2001.

[9] G. L. Sicuranza and A. Carini, "Nonlinear multichannel active noise control using partial updates," in Proceedings of IEEE International Conference on Acoustics, Speech, and Signal Processing (ICASSP '05), vol. 3, pp. 109-112, Philadelphia, Pa, USA, March 2005.

[10] E. Bjarnason, "Analysis of the filtered-X LMS algorithm," IEEE Transactions on Speech and Audio Processing, vol. 3, no. 6, pp. 504-514, 1995.

[11] O. J. Tobias, J. C. M. Bermudez, and N. J. Bershad, "Mean weight behavior of the filtered-X LMS algorithm," IEEE Transactions on Signal Processing, vol. 48, no. 4, pp. 1061-1075, 2000. 
[12] H.-C. Shin and A. H. Sayed, "Mean-square performance of a family of affine projection algorithms," IEEE Transactions on Signal Processing, vol. 52, no. 1, pp. 90-102, 2004.

[13] M. Bouchard and S. Quednau, "Multichannel recursive-leastsquares algorithms and fast-transversal-filter algorithms for active noise control and sound reproduction systems," IEEE Transactions on Speech and Audio Processing, vol. 8, no. 5, pp. 606-618, 2000.

[14] V. J. Mathews and G. L. Sicuranza, Polynomial Signal Processing, John Wiley \& Sons, New York, NY, USA, 2000.

[15] P. Strauch and B. Mulgrew, "Active control of nonlinear noise processes in a linear duct," IEEE Transactions on Signal Processing, vol. 46, no. 9, pp. 2404-2412, 1998.

[16] D. P. Das and G. Panda, "Active mitigation of nonlinear noise processes using a novel filtered-s LMS algorithm," IEEE Transactions on Speech and Audio Processing, vol. 12, no. 3, pp. 313322, 2004.

[17] S. J. Elliott, I. Stothers, and P. A. Nelson, "A multiple error LMS algorithm and its application to the active control of sound and vibration," IEEE Transactions on Acoustics, Speech, and Signal Processing, vol. 35, no. 10, pp. 1423-1434, 1987.

[18] A. H. Sayed, Fundamentals of Adaptive Filtering, John Wiley \& Sons, New York, NY, USA, 2003.

[19] T. Y. Al-Naffouri and A. H. Sayed, "Transient analysis of datanormalized adaptive filters," IEEE Transactions on Signal Processing, vol. 51, no. 3, pp. 639-652, 2003.

[20] S. Haykin, Adaptive Filter Theory, Prentice-Hall, Englewood Cliffs, NJ, USA, 2002.

[21] L. Tan and J. Jiang, "Adaptive Volterra filters for active control of nonlinear noise processes," IEEE Transactions on Signal Processing, vol. 49, no. 8, pp. 1667-1676, 2001. 